\title{
Design and development of a learning progression about stellar structure and evolution
}

\author{
Arturo Colantonio and Silvia Galano \\ Physics Division, School of Science and Technology, University of Camerino, 62032, Camerino, Italy \\ Silvio Leccia \\ Liceo Scientifico R. Cartesio, Giugliano, 80014, Naples, Italy \\ Emanuella Puddu \\ INAF, Astronomical Observatory of Capodimonte, 80138, Naples, Italy \\ Italo Testa* \\ Department of Physics "E. Pancini", University Federico II, 80126, Naples, Italy
}

(Received 13 May 2017; published 15 June 2018)

\begin{abstract}
[This paper is part of the Focused Collection on Astronomy Education Research.] In this paper we discuss the design and development of a learning progression (LP) to describe and interpret students' understanding about stellar structure and evolution (SSE). The LP is built upon three content dimensions: hydrostatic equilibrium; composition and aggregation state; functioning and evolution. The data to build up the levels of the hypothetical LP (LP1) came from a 45-minute, seven-question interview, with 33 high school students previously taught about the topic. The questions were adapted from an existing multiple-choice instrument. Data were analyzed using Minstrell's "facets" approach. To assess the validity of LP1, we designed a twelve-hour teaching module featuring paper-and-pencil tasks and practical activities to estimate the stellar structure and evolution parameters. Twenty high school students were interviewed before and after the activities using the same interview protocol. Results informed a revision of LP1 (LP2) and, in parallel, of the module. The revised module included supplementary activities corresponding to changes made to LP1. We then assessed LP2 with 30 high school students through the same interview, submitted before and after the teaching intervention. A final version of the LP (LP3) was then developed drawing on students' emerging reasoning strategies. This paper contributes to research in science education by providing an example of the iterative development of the instruction required to support the student thinking that LPs' levels describe. Concerning astronomy education research, our findings can inform suitable instructional activities more responsive to students' reasoning strategies about stellar structure and evolution.
\end{abstract}

DOI: 10.1103/PhysRevPhysEducRes.14.010143

\section{INTRODUCTION}

To describe and interpret how students develop their understanding of a given concept across school levels, research in science education increasingly promotes the use of learning progressions (LPs) [1]. LPs are usually built around "big ideas" [2], namely, "core" discipline concepts that help students connect everyday phenomena, empirical laws, and explanatory models $[3,4]$. In

\footnotetext{
*italo.testa@unina.it

Published by the American Physical Society under the terms of the Creative Commons Attribution 4.0 International license. Further distribution of this work must maintain attribution to the author(s) and the published article's title, journal citation, and DOI.
}

astronomy, LPs have been developed and validated for celestial motion and Solar System formation. LPs about celestial motion address specifically change of seasons [5-7], Moon phases [8], motion of the Sun and of the Moon $[9,10]$. Earth and space perspective and the relationships between spatial and causal reasoning were adopted by our group to develop a unifying LP about seasonal changes, Moon phases, and solar and lunar eclipses [11]. Recently, a LP has been proposed about Solar System formation based on four interrelated dimensions: physical properties, dynamical properties, formation, and gravity [12]. While representing valuable efforts, such LPs cover only partially the richness of astronomy and astrophysics endeavors, especially for higher levels of education. For this reason, in this paper, we discuss a LP about stellar structure and evolution (SSE). 
SSE provides meaningful explanatory models for a broad range of phenomena in the field of astronomy [13]. First, drawing on SSE, the presence and production of heavy elements can be explained and justified, while the conditions under which life may develop in the Universe can be validated through astrobiology models. Second, stellar evolution is relevant since by studying and observing supernovae, galaxies, and galaxy clusters, it is possible to improve and validate models of the Universe, and hence our knowledge of its age, geometry, composition, and expansion. As an example, recent nearinfrared observations of Cepheid variables in host galaxies of the type Ia supernova led to an unpreceded accurate determination of the local value of the Hubble constant [14].

Apart from providing an explanatory framework for relevant astronomical phenomena, SSE is worth developing a learning progression for since it attains students' everyday experience. Students learn about stars starting from simple observations of the sky at night: such aspect warrants that it is possible to define a lower anchor of the LP starting from students' ideas. For instance, students learn since their childhood that the Sun, the closest star to Earth, is essential for many biological and physical processes on Earth involving human, animals, and plants. Moreover, stars can indicate different times of the year since some of them rise in different hours in winter and summer. In addition, using simple observations with telescopes, students can distinguish between stars and planets and even estimate a star's age and temperature. Third, since emitted light is the only mean to gain insight about the nature of stellar structure, students' learning may be focused on spectral analysis, which is especially important in astrophysics research. In such a way, students can be familiarized with an indirect inquiry methodology used in science when a system is not directly accessible or reproducible in a laboratory. Finally, when dealing with SSE, students apply core concepts in classical and modern physics such as force, energy and heat conduction, light emission, propagation and interaction with matter, and atomic structure, thus connecting mechanics, thermodynamics, and nuclear physics.

The above arguments show that SSE meets the criteria proposed [8] to select big ideas in astronomy. However, while it may be a culturally fruitful and motivating context with the potential of increasing interest in science, research into students' understanding has been rather limited so far. The few studies in astronomy education research [15-18] show that most common misconceptions concern processes of stars' formation, the difference between stars and planets, and the distance between near stars and the Earth. Amongst these studies, only one [15] broadly deals with how different educational backgrounds affect students' knowledge about SSE. No other study so far identified qualitative distinctions across students' increasingly sophisticated knowledge levels and reasoning about SSE after curriculum instruction or when exposed to specific learning modules. The LP framework may be particularly suitable to address this dearth of research.

Therefore, in this paper, we detail our efforts to iteratively validate a tentative LP about SSE and the corresponding instructional activities. Our study was guided by the following question:

RQ: how do students progress in their understanding about SSE when exposed to iteratively designed instructional activities in a LP framework?

\section{THEORETICAL FRAMEWORK}

\section{A. Learning progressions}

The science education research community has often struggled to reach consensus on how to define and develop LPs [19-21]. Here we briefly discuss our understanding of LPs for the present study.

We adopted the following definition of LPs: "[LPs] are descriptions of the successively more sophisticated ways of thinking about a topic that can follow one another as children learn about and investigate a topic over a broad span of time" [22-24]. Rooted in a developmental view of learning [25-27], the LP framework assumes that students learn a given science content over an extended time period, starting from their intuitive ideas and progress through subsequent cognitive levels of a more sophisticated understanding of the topic [1]. The pattern of students' performance across the levels is determined by specific progress variables, which refer to specific aspects of students' knowledge and reasoning at a given level $[28,29]$.

The first level, usually labeled as lower anchor, may feature students' misconceptions, naïve explanations, or incorrect ideas about the topic. The intermediate levels correspond to more fully developed accounts or increasingly complex ideas, but incomplete or partially correct explanations may also be featured. The final level, upper anchor, corresponds to the accepted scientific explanation of the target concept, according to the chosen instructional level.

The LP framework allows us also to iteratively describe the interplay between the science content, the instructional methodologies, and assessment strategies used to investigate students' achievements. In particular, to demonstrate that students advance in their understanding about a given topic in the way hypothesized by a LP, researchers can design instructional activities that reflect student's ideas to support their hypothesis [30]. Correspondingly, LP levels are defined according to the specific learning objectives of the instructional activities [20]. When implementing the designed activities, empirical evidence about the different levels of the hypothetical LP is collected, so that the findings may inform a revision of the LP and align it with 
the actual students' achievements. Such a research-based cycle [31] ends when alignment between actual and hypothesized outcomes becomes sufficiently satisfactory.

Some authors have raised the issue of LP grain size [21]. Grain size of a LP can be understood as the shift in knowledge or reasoning that students need to make to move from a given level of the LP to the next one. LPs may feature coarse-grained or fine-grained levels, according to scope and learning objectives. The decision about grain size affects assessment of the student performances. In particular, coarse-grained levels may require shifts in general reasoning and knowledge and are usually associated with large-scale assessments. LPs with fine-grained levels focus on more detailed descriptions of students' learning, emphasizing nuanced differences and critical elements of change between students' performances at different levels. We chose a fine-grained design because we wanted to (i) identify qualitative distinctions between increasingly sophisticated knowledge and reasoning and (ii) iteratively develop a classroom teaching intervention to help students moving between the levels of our LP.

\section{B. Students' difficulties about SSE}

As stated in the introduction, research into students' understanding about SSE is rather limited [32]. Most of the results emerge from more general studies about students' conceptions in astronomy. Earlier studies [17] showed that students (i) do not distinguish between stars and planet, (ii) think that the Milky Way is composed by stars that are very close each other, (iii) struggle in estimating distances between stars and Earth, and (iv) think that stars are motionless celestial objects.

Most recent studies show that students rarely explain SSE in a detailed way. As far as the formation of a star is concerned, students often do not recognize the role of gravity: for instance, in one of the studies described [33] only about $16 \%$ of students referred to gravity as the cause for collapse of a nebula, from which the star is formed. Moreover, in some cases, students were not able to relate the spherical form of a star to gravity being a central force.

Concerning the functioning of a star, one study [15] showed that students think that a star is a "burning object," which releases some kind of "energy;" however, students find it difficult to understand how such energy is produced within a star. For instance, nuclear reactions, which are mainly responsible for stars' inner physical processes (expansion, compression, temperature variations), are often confused with chemical reactions, and, only rarely, students exploit thermodynamics laws to describe a star's inner processes.

To this concern, in other cases, students think that the bigger the radius of a star, the bigger its mass, thus misinterpreting its role in the evolution of the star [33]. Consequently, students may wrongly interpret the Hertzsprung-Russel (HR) diagram as a trajectory or a star's position in a "universal" space vs time graph. Finally, some students think that a star emits only monochromatic light [15].

\section{METHODS}

\section{A. Instructional context of the study}

The teaching of SSE in the Italian Earth Sciences curriculum targets students at the beginning of the high school. At this level, students are supposed to know only some basic notions about the heliocentric model of solar system and astronomical phenomena, hence no physics background is required. The earth sciences course is generally an introduction to the Universe and Earth motions, with emphasis on basic astronomy phenomena such as seasons or Moon phases. We informally know from teachers participating in our in-service training courses that SSE is only qualitatively addressed. However, to better describe the extent to which SSE is covered, we looked at a typical textbook treatment of the topic [34]. Two chapters (out of 16) are devoted to the main properties (e.g., composition, color, evolution) of the stars (and of the Sun in particular). According to teachers' experience, usual teaching time spent for the two chapters is about four to ten hours, depending on the teacher.

The chapter that concerns SSE includes the following subtopics: celestial objects, the celestial sphere and the stars' position, the life of stars, the HR diagram, galaxies, origin of the Universe. Stars are presented as rotating celestial objects formed by gases, mostly hydrogen and helium, and other chemical elements, but no account is given for how these elements, which constitute a star, are created. Birth, life, and death of stars are described in terms of different phases, which can be graphically represented by the HR diagram. The mass is mentioned as the main factor that influences a star's life and evolution.

Then, it is remarked that a star is an object that produces light and that the emitted light depends on the temperature at the surface: stars with a surface temperature of $10^{4} \mathrm{~K}$ order are the blue ones, while stars with surface temperature of about $10^{3} \mathrm{~K}$ are the red ones.

The birth of a star is qualitatively described as an "aggregation" of fine dust and cold gases, which are diffused around the cosmic space. Gravitational collapse is introduced to justify the heating of the inner gases to give rise to the nuclear reactions, which transform hydrogen into helium and release energy in form of heat. The energy viewpoint is thus adopted to justify in a qualitative way the stability of a star. Nuclear reactions are not addressed in the earth sciences syllabus, but in the math and physics one, at the end of the fifth year of high school. In particular, SSE is used as a teaching context for hydrogen nuclear fusion, presented as the transformation of four protons into a helium nucleus. Finally, composition of the stars is described in terms of plasma. 


\section{B. Choice of knowledge dimensions}

As we sought to develop the hypothetical initial LP (LP1) about the SSE, we took into account both Italian high school curriculum and literature in astronomy education. As described in the previous paragraph, Italian earth sciences curriculum teaching focuses on a qualitative description of SSE. Underlying physical concepts are only addressed later during the curriculum. Given this separation, we hence hypothesized that most of the difficulties that students of our population found when learning about SSE might be concerned with physics mechanisms at their basis. This hypothesis is coherent with research findings, which show, for instance, that students struggle in recognizing the role of gravity in stellar stability and confuse chemical and nuclear reactions. Because of their complexity, such mechanisms necessarily connect different physics content areas-classical mechanics, thermodynamics, nuclear physics, and matter structure. As a consequence, a hypothetical LP about SSE had to be multidimensional in order to preserve such connections.

Hence, drawing on accepted astrophysics models of stars, we chose three distinct but interrelated dimensions to describe students' knowledge about SSE: (i) hydrostatic equilibrium; (ii) composition and aggregation state; (iii) functioning and evolution. These dimensions combine relevant ideas and models that explain the complex variety of phenomena related to SSE (stability, composition, formation, functioning, death). Moreover, they harness in an interconnected way the two most relevant physics concepts-namely, gravity and nuclear reactions-that are necessary to explain SSE. Finally, the three dimensions include topics that are taught in the earth sciences and math and physics curricula and about which students show many misconceptions. Consequently, the three dimensions encompass also students' ideas and models, as reported in the literature.

However, while curriculum materials, astronomy education research studies, and astrophysics models were a useful starting point to identify knowledge dimensions, to increase reliability in the development of LP1, we decided to derive empirically the progress variables and students' levels of achievement.

\section{Instrument}

To develop the levels of LP1 for the three identified dimensions, we used an approximately 45-minute, sevenquestion interview (see Appendix A for the complete protocol). The general topics of the questions (e.g., composition and formation of a star, forces involved in hydrostatic equilibrium) were drawn from an existing multiple-choice instrument [32], but questions were purposely designed for the present study in an interview format. We retained only the first question "What is a star"? since its formulation was well suited to such a format. We remark that we chose as a research tool the interview since we were interested in unfolding students' reasoning strategies, which could be not limited to topics addressed in previous questionnaires or suitably fit in predetermined schemes.

Students' knowledge about each chosen dimension was probed with three questions, except for the hydrostatic equilibrium, which was addressed in only one specific question (Table I). The reason was that this question targeted forces involved in stellar stability and we expected it to be general enough to elicit during the discussion most of the students' ideas about the topic. The complete protocol reports more details.

\section{Sample}

Three samples were involved at the various stages of the study, which lasted three years. To develop LP1, we interviewed 77 high school students, from 13 to 18 years old (sample 1). Thirty students (S1-S30) were attending the first year of the same stream. Forty-seven students (S31-S77) came from two classes at the end of their high school stream. This sample was considered representative of Italian high school students since, along the five years of instruction, they had been taught about SSE with a typical earth sciences curriculum-based textbook and were expected to have been taught also about the involved physics concepts in the math and physics courses.

TABLE I. Correspondence between knowledge dimension of the LP about SSE and the interview questions. Level mapping for LP1 is also reported (see Section III. E. for details). The complete mapping is reported in Supplemental Material I [35].

SSE knowledge dimensions

\begin{tabular}{|c|c|c|c|c|c|c|c|}
\hline \multirow[b]{2}{*}{ LP1 levels } & \multirow{2}{*}{$\frac{\begin{array}{l}\text { Hydrostatic } \\
\text { equilibrium }\end{array}}{\text { Q4 }}$} & \multicolumn{3}{|c|}{ Composition and aggregation state } & \multicolumn{3}{|c|}{ Functioning and evolution } \\
\hline & & Q1 & Q2 & Q3 & Q5 & Q6 & Q7 \\
\hline Upper Anchor & Scientific & Scientific & Scientific & Scientific & Scientific & Scientific & Scientific \\
\hline Level 2 & Partial & At least partial & At least partial & At least partial & At least partial & At least partial & At least partial \\
\hline Level 1 & Partial & At least partial & At least partial & Naïve & At least partial & At least partial & Naïve \\
\hline \multirow[t]{3}{*}{ Lower anchor } & Naïve & Naïve & Naïve & Naïve & Naïve & Naïve & Naïve \\
\hline & & Naïve & Any & Any & Naïve & Any & Any \\
\hline & & Any & Naïve & Any & Any & Naiive & Any \\
\hline
\end{tabular}


Such a choice of a sample of students warranted us the possibility to collect empirical evidence for all levels of LP1. We expected that the distribution of students across the levels of LP1 should reflect their background knowledge acquired in earth sciences and physics; in particular, students at the end of the course (17-18 years old) should be located at the upper levels of LP1.

Subsequent revised versions of LP1 were assessed with two further samples: 20 high school students (17-18 years old, sample 2, S78-S97) participating to an extracurricular program organized at our physics department; 30 high school students (17-18 years old, sample 3, S98-S127) who were exposed to the revised teaching intervention during regular class activities. Given the emphasis on physics mechanisms underlying SSE, Sample 2 and 3 were purposely chosen amongst students who might be expected to be more motivated to learn about advanced physics topics. Moreover, we purposely chose to focus on 17-18 year old students after the first trial of the hypothetical LP, since we found challenges in developing LP levels building on existing literature, especially as far as the higher levels and upper anchor were concerned.

\section{E. Data analysis}

Interview data were analyzed using Minstrell's "facets" approach [36], which has been previously used to interpret students' reasoning in physics [37] and chemistry [20]. A facet has been defined as "the procedure that represents students' thinking as they attempt to explain situations or solve problems" [38]. As such, facets can be related to students' reasoning strategies and levels of understanding of the addressed concept. In our case, the choice of the facets analysis was mainly related to our decision to develop a finegrained LP, since facets may describe in great detail elements of students' reasoning and levels of knowledge, from more naïve ideas to targeted scientific understanding.

In our study, facets were first extracted from the interview data by two independent researchers using iteratively a constant comparative method [39]. Two professional astrophysicists coded them as naïve, partial, or scientific views, which correspond to incorrect ideas or known misconceptions, correct yet incomplete ideas, and correct ideas, respectively. Reliability was assessed through evaluation of inter-rater reliability. For sample 1, Cohen's kappa was 0.88 .

Then, for each dimension, emerging facets were mapped into levels of LP1 (Fig. 1 and Table I). The process was straightforward for the hydrostatic equilibrium dimension, because this dimension was probed by only one question: naïve views were mapped into the lower anchor; scientific views were mapped into the upper anchor; partial views were mapped into intermediate levels of the LP1, according to the extent to which they represented increasingly sophisticated reasoning. For the composition or aggregation state and functioning or evolution dimensions, each

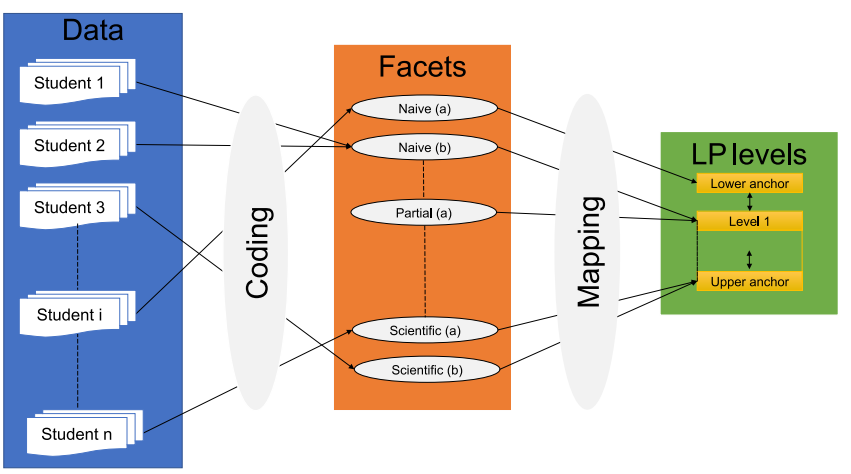

FIG. 1. Process of data analysis using the facets approach adopted in this study.

probed through three questions, the process, for each student, was as follows: first, we went through their answers and identified all obtained facets; then, the facets were mapped into suitable levels by grouping them according to the codes (naïve, partial, scientific). With such a process, we located each student into a level for each dimension based on the coding of the facets emerging from their answers. For instance, consider the dimension composition and aggregation state. Since we had three questions for this dimension, using our three-level coding system, we could have 27 combinations. The easiest to map were those corresponding to three answers coded as naïve or as scientific. In the first case, the student was assigned to the lower anchor, in the latter to the upper anchor. Of the remaining 25 combinations, if one facet corresponding to either question Q1 or Q2 was coded as naïve (14 combinations), the student was assigned to the lower anchor independently of their answer to question Q3, since they plausibly had an insufficient knowledge to discuss the basics about the nature of stellar composition. Clearly, a combination of facets as naïve-naïve-scientific is highly unlikely, if not impossible, since it would imply that a student has a correct model of the shape of a star and of its internal structure but shows some misconception about the nature of stars as celestial objects. Second, if the facets corresponding to both Q1 and Q2 were coded at least as partial and Q3 still as naïve (4 combinations), the student was assigned to L1, since ideas about the stellar inner structure and state of aggregation are still incorrect. Next, if the facet relative to question Q3 was coded at least as partial, and the facet corresponding to one of the questions Q1 and Q2 was coded at least as partial (7 combinations), the student was assigned to L2 since, with respect to L1, the shape of the stars is at least recognized, although not fully justified. The combinations of facets for level mapping are reported in Table I. The codebook for the construction of levels of the three dimensions from emerging facets, including facets categorization, representative students' excerpts, and the corresponding level of LP1 is available as Supplemental Material 1 [35]. 
Once LP1 was developed, we designed a first version of a teaching module (TLS1) featuring paper and pencil and practical activities to assess the validity of LP1. Before and after the implementation of TLS1, students of sample 2 were interviewed with the same protocol. The same analysis described above was carried out (Cohen's kappa for sample 2: 0.94). Then, facets were mapped into LP levels and results informed a revision to LP1. The resulting revised LP (LP2) was assessed thorough implementation of a new version of TLS1 (TLS2). TLS2 was revised using the outcomes of the TLS1 implementation. The worksheets used in the paper and pencil and practical activities of TLS1 and TLS2 are reported as Supplemental Material 2 [35].

The same interview protocol and the same data analysis and reliability measure (Cohen's kappa for sample 3: 0.95) were used. A final version of the LP (LP3) was then developed drawing on students' emerging learning outcomes.

The independency of students' samples, with respect to the initial distribution across the levels of LP1 and LP2, was tested through chi-square analysis. The overall sample size was sufficient to meet the assumptions for the use of this statistical test. Differently, given the limited number of students involved in subsequent implementations, distribution across the levels of LP2 and LP3 before and after TLS1 and TLS2 was analyzed through the nonparametrical test of marginal homogeneity. In a contingency table, marginal homogeneity refers to the lack of significant difference between one or more of the row marginal proportions and the corresponding column proportion. Marginal homogeneity occurs when the row totals are equal to the column totals. If the rows and the columns of the table contain a pre- and post-treatment distribution of a variable, a common interpretation to a marginal homogeneity is the absence of or no effect of the treatment [40].

\section{FINDINGS}

\section{A. Development of the hypothetical LP levels}

Levels of each of the three dimensions of LP1 are described in Table II. For the lower anchor, we also indicate references to previous findings that extend or are confirmed by our data. Table III reports the distribution of students among the levels of the three dimensions. As expected, differences between the students at the beginning and at the end of their high school course are statistically significant for the hydrostatic equilibrium and functioning and evolution dimensions $\left(\chi^{2}=36.952, \quad \mathrm{df}=2, \quad p<10^{-4}\right.$; $\chi^{2}=6.346$, df $=1, p=0.042$, respectively), but not for the composition and aggregation dimension $\left(\chi^{2}=3.428\right.$, $\mathrm{df}=3, p=0.330$ ). In the following, we present a description of students' facets for each dimension of the SSE big idea, by showing typical excerpts from the interviews.

Hydrostatic equilibrium.-Only a few students (4) showed a naïve view about a star's equilibrium.
S14 (14 years old): "A star is not in equilibrium since it gets smaller and smaller during its life"

The majority of 13-14 years old students (25 out 30 ) was not able to justify stars' equilibrium

S06 (14 years old): "Yes, I think that a star is in equilibrium"

I: "can you give me some justification for such equilibrium?"

S06: "well... I don't know... maybe because they don't move..."

A possible justification could be a scarce knowledge about the forces involved in circular motion. The great majority of $17-18$ years old students (34 out 47 ) justified stars' equilibrium with an incomplete balance of gravitational force:

I: “...So a star is formed by gases, but is it a stable structure?"

S35 (18 years old): “...uhm... yes... I think so...

I: "Why do you think so?"

S35: "well... I think that it is because of the gravitational force, which attracts the gases toward the center of the star, and of the centripetal force..."

I: "can you make a drawing for a small volume element?"

S35: "uhm... yes..." (see Fig. 2)

I: "can you explain your drawing?"

S35: "yes... the curved arrow is the centripetal force... the force due to rotation...the right arrow is the gravitational force towards the center of the Sun... and it's all because of the high temperature of the gas..."

I: "Can you explain such role of the temperature?"

S35: “...well, when you melt a particle with another one, they unite... they solidify... so, it's like soldering..."

I: "what would happen if there was no high temperature?"

S35: "... gases would be free to move and so the star would be not in equilibrium..."

The above excerpts show that the main difficulty for the student was to apply Newton's second law to the small rotating star's volume element. While he knew that a star is a celestial body in equilibrium, he struggled in the attempt to find out some sort of mechanism that could link all available pieces of knowledge, namely, gravity, rotation, and high temperature in the core. Only a few students (three out of 34) attempted to give a justification of the star's equilibrium taking also into account inner processes:

S37 (17 years old): "I think that inside a star there is gravity and thermal energy of the plasma mass, which balance one another, so that the inner of a star is in equilibrium" (he draws a model of star, see Fig. 3)

I: "Are you sure?"

S37: "well, not so sure, but I've studied that energy emissions balance gravity"

I: "What do you mean by energy emissions?"

S37: "well... emission of radiation... yes... I think that nuclear reactions emit radiation..." 
TABLE II. Initial LP about SSE (LP1).

\begin{tabular}{|c|c|c|c|}
\hline Level & Hydrostatic equilibrium & Composition and aggregation state & Functioning and evolution \\
\hline $\begin{array}{l}\text { Upper } \\
\text { Anchor }\end{array}$ & $\begin{array}{l}\text { Equilibrium of a star is } \\
\text { justified by balancing } \\
\text { gravitational force with } \\
\text { radiative pressure forces } \\
\text { or other physical } \\
\text { quantities related to } \\
\text { nuclear reactions on a } \\
\text { star's element of volume }\end{array}$ & $\begin{array}{l}\text { Stars are considered as celestial objects } \\
\text { made mainly of } \mathrm{H} \text { and He and } \\
\text { described by specific physical } \\
\text { quantities (mass, temperature, radius). } \\
\text { Spherical shape is recognized and } \\
\text { justified. Internal structure and state of } \\
\text { aggregation are recognized and } \\
\text { justified }\end{array}$ & $\begin{array}{l}\text { The role of gravity as a central force in } \\
\text { stars' formation is correctly } \\
\text { recognized. Stars' functioning is } \\
\text { justified in terms of nuclear reactions } \\
\text { and heat transfer mechanisms. Life and } \\
\text { death stages of a star are related to the } \\
\text { star's initial mass }\end{array}$ \\
\hline Level 2 & $\begin{array}{l}\text { Star equilibrium is justified } \\
\text { by an incomplete or } \\
\text { incorrect balancing of } \\
\text { forces, or other quantities } \\
\text { (e.g. energy) are } \\
\text { incorrectly involved }\end{array}$ & $\begin{array}{l}\text { Stars are considered as celestial objects } \\
\text { made mainly of } \mathrm{H} \text { and He and } \\
\text { described by specific physical } \\
\text { quantities (mass, temperature, radius). } \\
\text { Spherical shape is recognized but not } \\
\text { justified. Internal structure and state of } \\
\text { aggregation are not recognized or } \\
\text { correctly indicated. }\end{array}$ & $\begin{array}{l}\text { Gravity is recognized as attractive force } \\
\text { but its role in star's birth is unclear. } \\
\text { Stars' functioning is justified in terms } \\
\text { of nuclear reactions. The role of mass } \\
\text { in subsequent stages of the star's life, } \\
\text { including death, is unclear }\end{array}$ \\
\hline Level 1 & $\begin{array}{l}\text { Star equilibrium is } \\
\text { recognized but no } \\
\text { justification in terms of } \\
\text { forces is given }\end{array}$ & $\begin{array}{l}\text { Stars are considered as celestial objects } \\
\text { made of gases, in particular } \mathrm{H} \text { and } \mathrm{He} \text {. } \\
\text { Spherical shape is not recognized or } \\
\text { incorrectly justified. Internal structure } \\
\text { and state of aggregation are not } \\
\text { recognized or correctly indicated. }\end{array}$ & $\begin{array}{l}\text { Star's formation is attributed to a generic } \\
\text { attraction. The role of nuclear reactions } \\
\text { in the functioning of a star is } \\
\text { recognized but their role in the stages } \\
\text { of the star's life is unclear or incorrect. } \\
\text { Final stages of star's life are incorrectly } \\
\text { described }\end{array}$ \\
\hline $\begin{array}{l}\text { Lower } \\
\text { Anchor }\end{array}$ & $\begin{array}{l}\text { A star is considered as } \\
\text { a system not in } \\
\text { equilibrium [33] }\end{array}$ & $\begin{array}{l}\text { Stars are considered as solid celestial } \\
\text { objects made of dust, gases or pieces of } \\
\text { planets, or seen as the result of } \\
\text { collisions of other celestial bodies [15]. } \\
\mathrm{H} \text { and He can be recognized as } \\
\text { components, but not the main ones. } \\
\text { Shape is not recognized as regular [18] }\end{array}$ & $\begin{array}{l}\text { Gravity is not recognized in the formation } \\
\text { process [33]. Nuclear reactions are } \\
\text { recognized as important for stars' } \\
\text { functioning without explanation or } \\
\text { they are confused with "burning" } \\
\text { chemical reactions [15]. Final stages of } \\
\text { star's life are incorrectly described [33] }\end{array}$ \\
\hline
\end{tabular}

As a result, for LP1 we identified four levels of achievement that track students' understanding from a nonequilibrium view of stars towards a force-based model. Between the lower and upper anchors, level 1 describes a view in which the equilibrium is recognized but not justified, while level 2 represents a more developed transitional view of equilibrium in terms of an incorrect balance of forces. However, we note that no student was able to justify with a scientific view the stars' equilibrium. Hence, the upper anchor of the dimension was developed considering only astrophysics models. To this concern, in our description of the upper anchor, we assumed that the product of the volume element mass times its centripetal acceleration is much smaller compared to gravitational force and radiative pressure forces related to nuclear reactions. However, given its relevance from the education viewpoint, we decided to help students justify such assumptions in the teaching sequence. In particular, we tried to address the "counterbalancing" conception of circular motion with a computerbased task in the first teaching sequence. Finally, we

TABLE III. Sample 1 students' distribution across LP1 levels.

\begin{tabular}{|c|c|c|c|c|c|c|}
\hline \multirow[b]{2}{*}{ Level } & \multicolumn{2}{|c|}{ Hydrostatic equilibrium } & \multicolumn{2}{|c|}{ Composition and aggregation state } & \multicolumn{2}{|c|}{ Functioning and evolution } \\
\hline & $13-14 \mathrm{yr}$ & $17-18 \mathrm{yr}$ & $13-14 \mathrm{yr}$ & $17-18 \mathrm{yr}$ & $13-14 \mathrm{yr}$ & $17-18 \mathrm{yr}$ \\
\hline Upper anchor & 0 & 0 & 0 & 1 & 0 & 0 \\
\hline Level 2 & 1 & 34 & 6 & 5 & 0 & 4 \\
\hline Level 1 & 25 & 13 & 12 & 27 & 10 & 24 \\
\hline Lower anchor & 4 & 0 & 12 & 14 & 20 & 19 \\
\hline Total & 30 & 47 & 30 & 47 & 30 & 47 \\
\hline
\end{tabular}




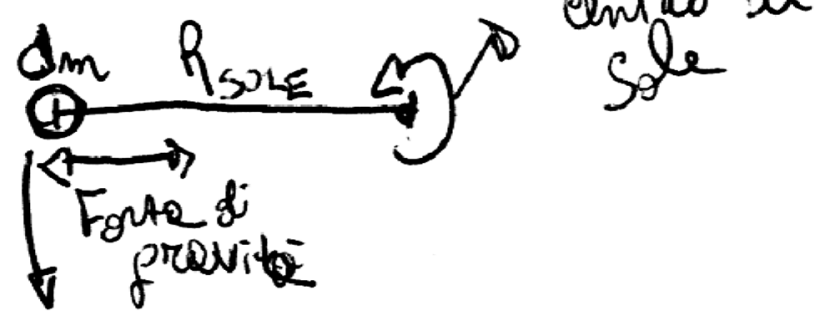

FIG. 2. Student's sketch of the forces acting on a star's small volume element. Translation of Italian text, from left to right: Gravitational force, $R_{\text {sun }}$, center of the Sun.

decided to not refer to centripetal or centrifugal force in the description of the LP levels, since such terms may cause misconceptions [41-44], as our data suggest.

Composition and aggregation state dimension.Overall, 26 out of 77 students held the incorrect view that stars are made of dust or small particles that are present in the Universe. The majority of students (51 out 77) held correct but incomplete views: six students claimed generically that stars are formed by gases, 45 explicitly named $\mathrm{H}$ and $\mathrm{He}$ as main components of a star, but only one student was able to include in his description other elements that are produced by a star. Overall, students held several misconceptions about chemical composition and states of matter within a star. For example,

I: "what happens at initial stage of the life of a star?"

S43 (17 years old): "Well... the star is born from a mass of gases, in which the elements begin to interact between them"

I: "what are these elements?"

S43: "mostly, $\mathrm{H}$ and He.."

I: ".. and how they interact?"

S43: "they are melt together releasing energy"

The following excerpts show the typical misconception that combustion processes happen within a star:

I: "what is a star?"

S21 (14 years old): "it's a celestial body formed by matter, at the state of plasma, with a certain temperature and mass

I: "and... how would you describe the composition of a star?"

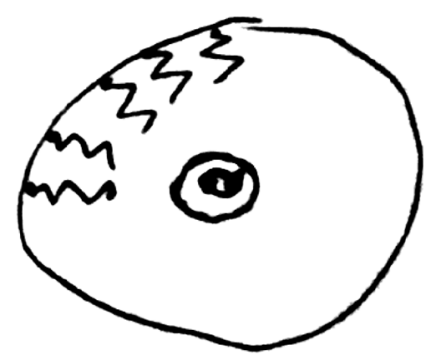

FIG. 3. Student' sketch of inner processes within a star.

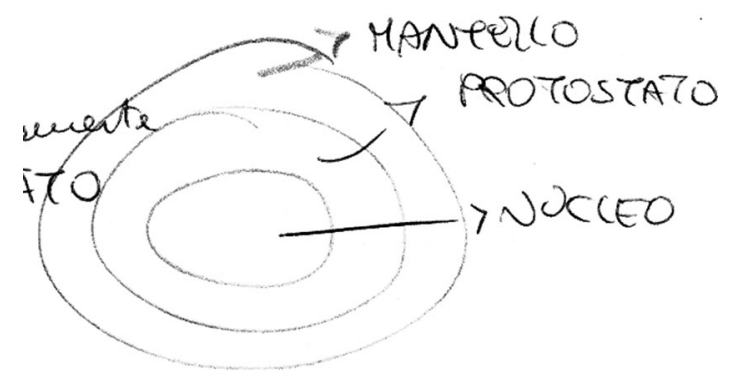

FIG. 4. Typical naïve student sketch of a star's internal structure. Translation of Italian labels, from top to bottom: mantle, first layer, core.

S21: "most of the matter is represented by $\mathrm{H}$ and $\mathrm{He} . . . "$ I: "Can you describe their aggregation state?"

S21: "... they have very high temperature... so through their combustion the star produces heat..."

Eleven students partially described also the internal structure of a star (see Fig. 4 for a typical drawing). Such structure may resemble the Earth's internal structure.

Only one student was slightly more precise:

S60 (17 years old): "A star is made at the center by a nucleus, in which the nuclear fusion reactions happen, and various layers; the outermost layer is the chromosphere, followed by the photosphere, the radiative zone and the convective zone..." (see Fig. 5).

Overall, the lower anchor of this dimension represents students' accounts of stars as solid objects, a view that seem to be related to a scarce knowledge about chemical elements and states of matter. Levels 1 and 2 represent more correct accounts based on the $\mathrm{H}-\mathrm{He}$ composition of stars, while shape and internal structure are still not clear. The upper anchor represents the fully developed scientific view based on $\mathrm{H}-\mathrm{He}$ composition and variable density inside the star.

Functioning and evolution dimension.-About half of the students (39 out of 77) claimed that a star is a celestial body born from a nebula, though through an unclear mechanism, and that during its life it gets smaller, colder, or darker until it dies.

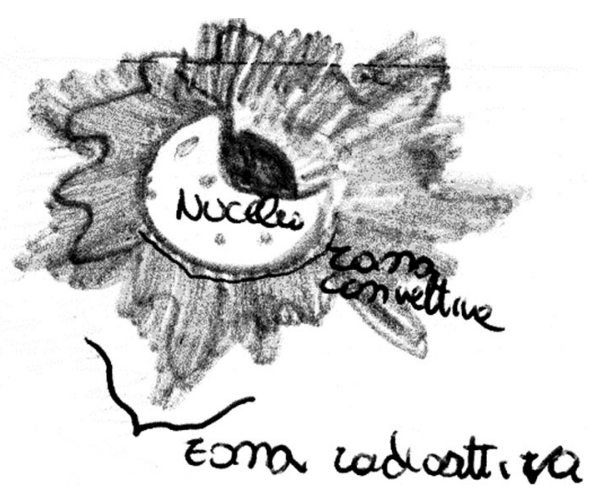

FIG. 5. More precise student sketch of a star's internal and external structure. Translation of Italian labels, from top to bottom: core, convective zone, radiative zone. 
Twenty students justified their reasoning through a model of nuclear reactions that "consume $\mathrm{H}$ and He until the star becomes a black hole". The following excerpts exemplify this "consuming" model:

I: "So, how would you describe initial and final stages in a star's life?"

S37 (17 years old): "In the initial stage, a star has a larger size, but as time goes by, $\mathrm{H}$ is used up and hence the star gets smaller until the final stages in which it explodes and becomes a supernova..."

I: "can you explain in more detail what happens in the final stages of a star's life?"

S37: "When the star is in its final stage, its gases, especially $\mathrm{H}$ are almost used up... So, the star emits a bright light... But, as gases are almost used up star becomes darker and darker and smaller until it snuffs... Or it explodes, as a supernova..." (he draws what he thinks are the different stages of a star, see Fig. 6).

Such underlying reasoning confuses collapse due to gravity with the consumption of matter.

The remaining students (38) attempted to relate composition and brightness of a star to internal nuclear reactions. Ten students related emitted visible light to nuclear processes, though showing incorrect ideas about the temperature of a star:

S64 (17 years old): "A star to shine must reach a temperature of 1 million degrees..."

Twenty-five students explicitly stated that nuclear reactions inside the star transform $\mathrm{H}$ into $\mathrm{He}$, but they often confused nuclear and chemical reactions or thermodynamics transformations:

S19 (14 years old) "... within a star, He is produced through heating of the $\mathrm{H}$ by the nuclear reactions"

One student attempted, at least to some extent, to give a mechanism for such transformation:

I: "... when does $\mathrm{H}$ is transformed into He?"

S54 (18 years old): "when the star reaches a very high temperature... that's when nuclear reactions within star's core begin to take place..."

I: "Yes... so these nuclear reactions transform $\mathrm{H}$ into He?"

S54: "Yes, $\mathrm{H}$ is transformed in He... Two nuclei are fused together thanks to the high velocity at which they

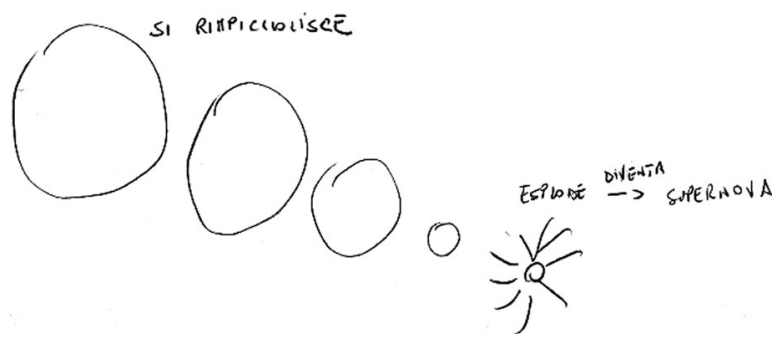

FIG. 6. Student sketch of a star's life stages. Translation of Italian labels, from left to right: [it] becomes smaller, explodesbecomes supernova. travel... big amounts of thermal and luminous energy are released because part of $\mathrm{H}$ mass is lost..."

Overall, for this dimension, the lower anchor represents naïve accounts of stars' functioning and evolution, with no reference to gravity or nuclear reactions. The pattern from level 1 through the upper anchor represents increasingly complex students' reasoning based on gravity, thermodynamics, and energy-binding considerations. We note that, also for this dimension, we had to develop the upper anchor of LP1, referring solely to advanced astrophysics models.

\section{B. Development of TLS1 about SSE}

Dimensions and levels of LP1 informed the design of TLS1. In the following, we give a brief description of the module phases. To ensure a more coherent development of students' understanding of the SSE big idea, progress variables of the three dimensions were set as learning targets in each phase of the module. Moreover, we adopted in the activities of the module a guided inquiry approach, which was used also in previous studies by our group [45]. The aim was to let student engage in reasoning strategies that could help them move across the levels of LP1, depending on their starting level. More details about specific activities can be found in Ref. [46]. The total duration of this version of the module was $12 \mathrm{~h}$.

First phase ( $4 \mathrm{~h})$ : TLS1 activities begin by pointing out that stars are celestial objects that rotate, and that gravity is the main responsible for star's rotation, formation and shape. A justification of rotational motion in terms of conservation of angular momentum is also given.

Second phase $(4 \mathrm{~h})$ : students are guided to study the physical quantities that characterize a star. In particular, they first measure the frequency of Sun sound using an audio editor and derive the mathematical relationship between mass, temperature, and radius of a star [47]. The aim is not only to show how different stellar structure parameters are related to each other but also to familiarize students with methods used in astrophysics.

Then, assuming the shape of a star as spherical due to the action of gravity, students are introduced to the hydrostatic equilibrium of a star and measure the Sun's rotational speed with Tracker software (Fig. 7).

The aim is to compare the centripetal and gravitational acceleration of a small volume element to show that the product of mass times centripetal acceleration - centripetal force- has not the same magnitude of gravitational force, contrarily to what the majority of students might think. Hence, through the activity, we also address the typical students' misconception that circular motion implies the existence of a "centrifugal force" By noting that a standard star does not change its average radius over time, or that a star does not collapse, students might understand that the small volume element is subject to a normal force other than the gravitational force. 


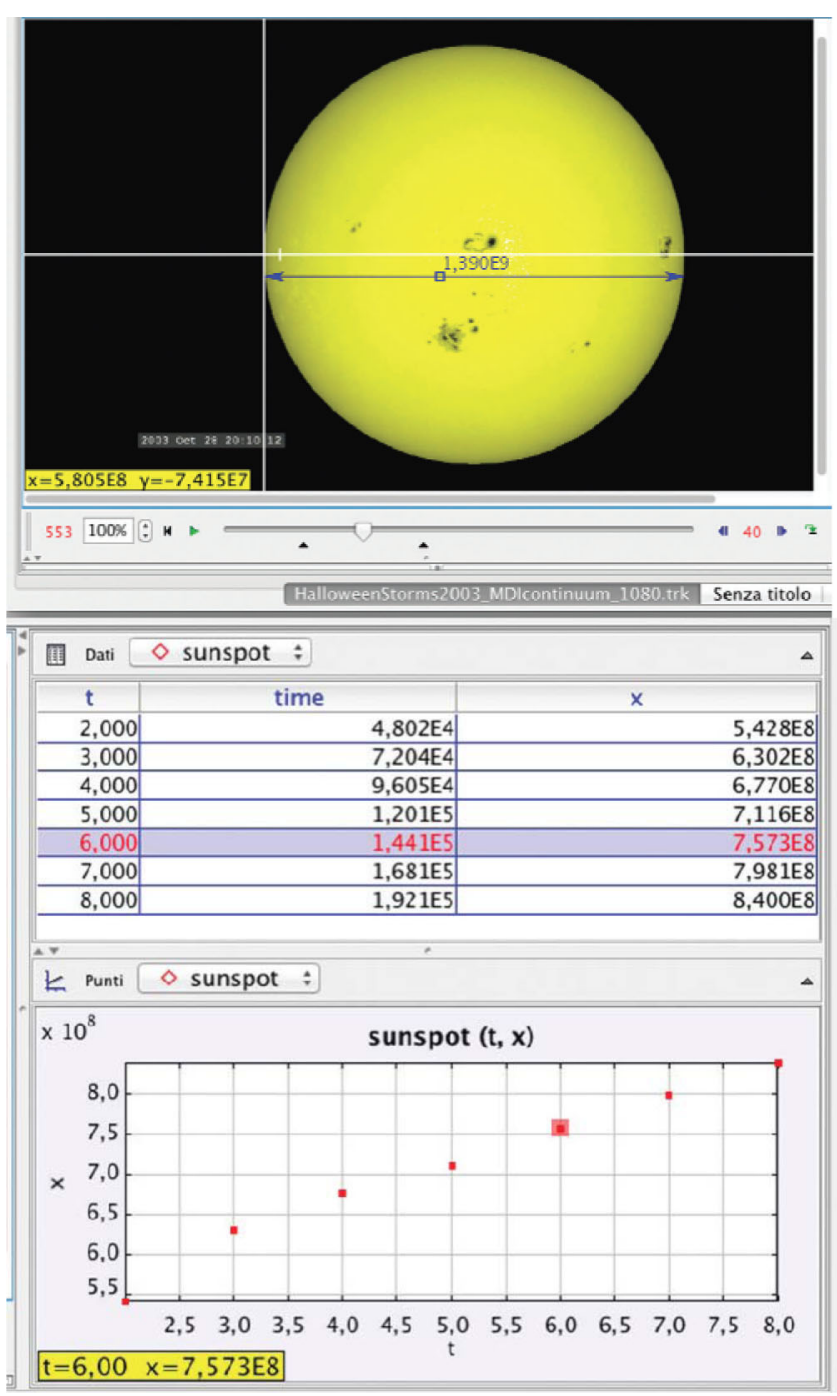

FIG. 7. Tracker screenshots on the measurement of Sun's rotational velocity.

Third phase $(4 \mathrm{~h})$ : Equilibrium condition for a star is interpreted through the physical mechanism of the production of energy in the core due to nuclear fusion reactions. Students' attention is then focused on pressure forces, which are used to justify a star's equilibrium.

To address the consuming view of nuclear reactions detected when developing LP1, the production of energy and neutrinos as a result of decrease in mass is emphasized as an important feature of nuclear reactions.

Hence, students may grasp that such reactions depend solely on the age and mass of the star, and they consist mostly of hydrogen fusion into helium for most of a star's life. Finally, stellar structure is introduced to describe where nuclear reactions take place in the star.

\section{Revision of hypothetical LP}

In the following, we report about the results of the implementation of TLS1 with sample 2. We detail how
TABLE IV. Sample 2 students' distribution across LP1 levels.

\begin{tabular}{|c|c|c|c|c|c|c|}
\hline \multirow[b]{2}{*}{ Level } & \multicolumn{2}{|c|}{$\begin{array}{l}\text { Hydrostatic } \\
\text { equilibrium }\end{array}$} & \multicolumn{2}{|c|}{$\begin{array}{l}\text { Composition and } \\
\text { aggregation state }\end{array}$} & \multicolumn{2}{|c|}{$\begin{array}{l}\text { Functioning } \\
\text { and evolution }\end{array}$} \\
\hline & Pre & Post & Pre & Post & Pre & Post \\
\hline Upper anchor & 1 & 2 & 0 & 0 & 0 & 0 \\
\hline Level 2 & 12 & 18 & 2 & 6 & 0 & 2 \\
\hline Level 1 & 7 & 0 & 8 & 7 & 8 & 16 \\
\hline Lower anchor & 0 & 0 & 10 & 7 & 12 & 2 \\
\hline Total & 20 & 20 & 20 & 20 & 20 & 20 \\
\hline
\end{tabular}

specific students moved through the different levels of LP1 and how progressions relate to the instructional intervention. Then, we show how findings influenced changes to LP1 and to the teaching module. Analysis shows that preinstruction distribution of sample 2 students in the levels of LP1 (Table IV) was not significantly different to that of the 17-18 years old students of sample 1 for all dimensions $\left(\chi^{2}=2.914\right.$, $\mathrm{df}=2, p=0.233$; $\chi^{2}=2.849, \quad \mathrm{df}=3, \quad p=0.416 ; \quad \chi^{2}=2.162, \quad \mathrm{df}=1$, $p=0.141$, respectively). After the intervention, findings show a moderate but significant improvement in students' understanding about SSE (marginal homogeneity test: $p=0.005 ; p=0.008 ; p=0.001$, respectively). On average 9 students out of 20 improved their level across LP1.

Hydrostatic equilibrium.-Despite some improvement (8 out 20 students moved from a lower to an upper level of LP1), after the teaching intervention, overall 18 out of 20 students either (i) claimed that the only force acting on a small star volume element is the gravitational force or (ii) balanced gravity with other "forces," as, e.g., an unknown "normal" force. In both cases, pressure forces within the star are disregarded (level 2). For instance, S83 before the intervention was not able to justify why a star was in equilibrium (level 1). Earlier, he had been taught about this topic only in earth sciences subject.

S83: “... I don't know, but I think it's in equilibrium...." I: "why do you think that?"

S83: "well... uhm... it's difficult... because a star evolves..."

I: "why is it difficult?"

S83: “... in the sense that it is not possible for me to say with certainty if it is in equilibrium... maybe it is... but it changes its state many times during its life. First, there is the proto-star, and then it burns and may become a super nova or black hole..."

After the intervention, he was able to at least reason about equilibrium in terms of forces (level 2):

S83: "Yes... now I know the answer... a star is a structure in equilibrium, and the forces that interact are the gravitational force and the normal force"

I: "Are you sure? Can you sketch the forces acting on a small element of mass?" 


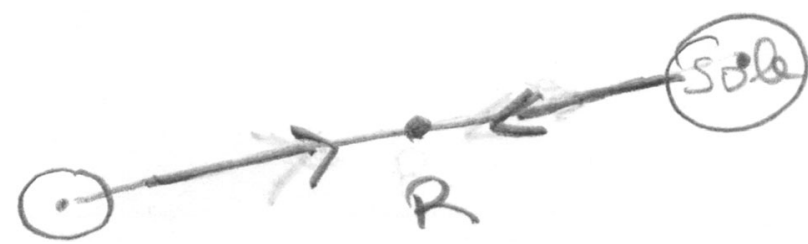

FIG. 8. Sample 2 student's sketch of the forces acting on a star's small volume element. Translation of Italian label: Sun.

S83: "... the two forces have the same direction..." (he draws the element of mass and the core of the Sun, Fig. 8).

I: "ok, can you calculate acceleration of the small mass?"

S83: "Yes... When we calculate the acceleration of gravity, we got... $0,2 \times 10^{3} \mathrm{~ms}^{-2}$, while for the normal acceleration we got... $0,5 \times 10^{-2} \mathrm{~ms}^{-2} \ldots$ "

I: "so what can you conclude?"

S83... (laughs) “... oh no, it is not in equilibrium! So, I was right...it's very difficult... we cannot know..."

Such excerpts suggest that the emphasis put during the second phase of TLS1 on the explicit calculation of centripetal acceleration to show that some other force other than gravity must be at play in a star, was not successful in helping students include somehow in their reasoning pressure forces. Only two students showed some improvements in their reasoning about forces in a star. For example, S87 before the intervention answered to the interview question as follows:

S87: "I think a star is in equilibrium because there is gravity..."

I: "what do you mean by gravity?"

S87: "...well, the gravity holds together the gases of the star... since they are light they are strongly attracted..."

I: "do you think that such a strong attraction could make the star collapse?"

S87: "no... it is not enough to make a star collapse... it is more like the force exerted on electrons by nucleus in the atom..." (level 2)

After the intervention, she was able at least to introduce a new physical quantity to justify equilibrium.

I: "So, now, do you think a star is in equilibrium?"

S87: "Yes... Forces that maintain it in equilibrium are the gravitational energy and the reaction of radiation..."

I: ".. uhm... what do you mean by "reaction" of radiation?"

S87: “... it's something inside the star... heat, thermal energy, luminous energy..." (Upper anchor).

While not formally correct, her reasoning suggests the correct view that some kind of internal process accounts for the force that balances gravity.

Overall, given our difficulty in categorizing students' answers that only partially encompass the gravity vs inner processes' balance, as well as the persistent difficulty students experienced in identifying relationships between forces in a star, two new levels after level 2 and before the upper anchor were introduced for this dimension: (i) a first one corresponding to the capability of evaluating magnitudes of centripetal and gravitational acceleration for the Sun using Newton's law; and (ii) a second one corresponding to the capability of using thermodynamics to qualitatively or partially justify stars' equilibrium.

Construction of these intermediate levels from facets is reported in the Supplemental Material 1 [35].

Composition and aggregation.-After the intervention, the majority of students (13) correctly claimed that stars are made of $\mathrm{H}$ and $\mathrm{He}$. Six of them made also a correct drawing of the star inner layers, but none of students was able to relate the internal structure to the chemical and physical composition of stars.

For instance, S93 initially claimed that the stars were made of dust and burning particles, with no internal structure. After the intervention, his answer shows no substantial difference:

I: "What is the composition of a star?"

S93: “...well... it is made of particles, from the core to the external layer..."

I: "how are they distributed? You can draw if you want..."

S93: (draws a model of star, see Fig. 9)

I: "Can you explain it?"

S93: "... in the nucleus there are particles that are denser... dust is compacted since the star gets smaller during its life..."

I: "How does this structure form?"

S93: "Through a process that lasts millions of years, a nebula is condensed forming an inner solid core... On its surface, there is a layer made of dust and gases where chemical reactions take place..."

Such reasoning suggested to us to put more emphasis on the continuity of the changes in the density inside the star.

Other students showed some improvement. S94 before the intervention confused the process of formation of the star with the mechanisms underlying its structure:

I: "What is the composition of a star?"

S94: "...it is made of $\mathrm{H}$ and He..."

I: "... And what is its shape and internal structure?"

S94: "...well... it is like a big ball of gases..."

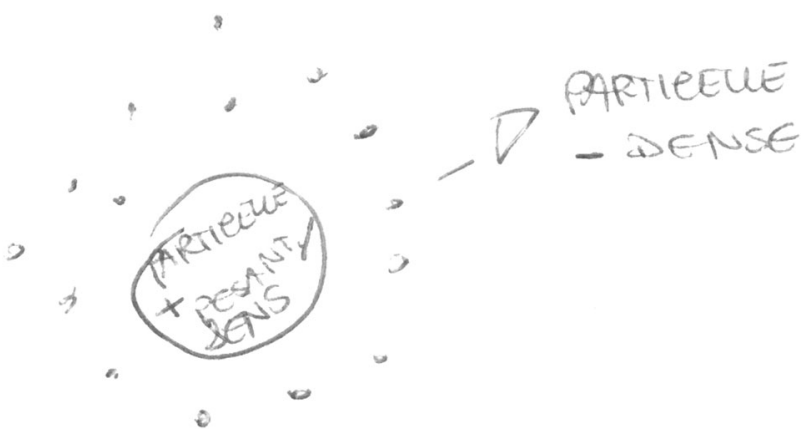

FIG. 9. Sample 2 student's sketch of mass distribution within a star. Translation of Italian labels, from left to right: higher density of particles, lower density of particles. 


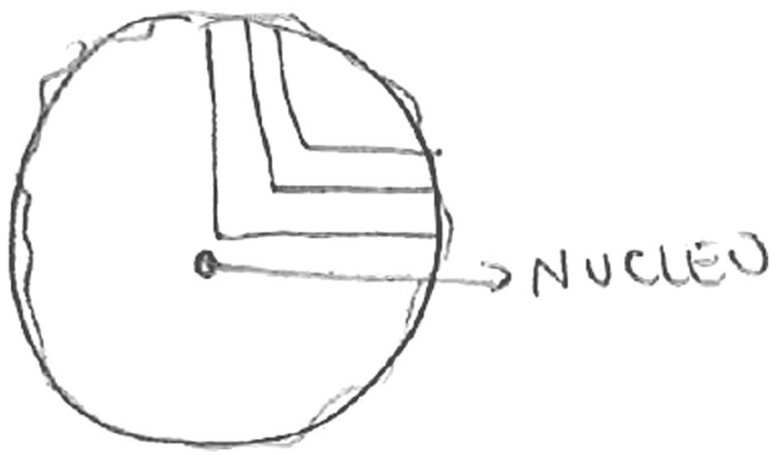

FIG. 10. Sample 2 student sketch of a star's internal structure. Translation of Italian label: nucleus.

I: "...How are they distributed?"

S94: "...I think that they are uniformly distributed..."

After the intervention, he showed a typical level 2 reasoning:

I: "What is the composition of a star?"

$\mathrm{S} 94$ "... a star is made of $\mathrm{H}$ and $\mathrm{He} . . .85 \%$ hydrogen, $10 \%$ Helium, traces of Oxygen..."

I: "And what is its shape and internal structure? You can make a drawing..."

S94 draws a circular star and its internal structure (see Fig. 10)
I: "ok, and are these lines the different layers inside a star?"

S94: "well, actually these are not so definite... I don't know... there is a convection zone, a radiative zone, a photosphere and chromosphere... the solar crown..."

I: "How are these layers related to chemical elements of the star?"

S94: "well... I don't know, for sure nuclear fusion reactions that generate $\mathrm{He}$ from $\mathrm{H}$ take place in the star's core ... and in the solar crown there's light..."

The excerpts show that this student still had difficulty in correctly identifying variable density inside a star. Consequently, for this dimension, TLS1 was only partially effective in helping students develop a mechanism to justify stars' composition.

Hence, given the difficulty in correctly identifying the chemical composition (e.g., the presence of elements) in the core and in the outer layers of a star, a new level between level 2 and the upper anchor of this dimension was introduced (Table V). This level refers to the capacity to account at least for a star's internal structure and state of aggregation, but without the reference to the density that varies as a function of the distance from the inner core to outer shells. The capability of correctly using variable density to describe a stars' internal structure was added to the upper anchor. The construction of the new level and the

TABLE V. Revised LP about SSE (LP2). Lower anchor to level 2 are as in LP1 (Table II).

\begin{tabular}{|c|c|c|c|}
\hline Level & Hydrostatic equilibrium & Composition and aggregation state & Functioning and evolution \\
\hline $\begin{array}{l}\text { Upper } \\
\text { Anchor }\end{array}$ & $\begin{array}{l}\text { Equilibrium of a star is } \\
\text { justified by balancing } \\
\text { gravitational force and } \\
\text { pressure forces, related to } \\
\text { nuclear reactions, on a } \\
\text { star's element of volume }\end{array}$ & $\begin{array}{l}\text { Stars are considered as celestial objects made } \\
\text { mainly of } \mathrm{H} \text { and He and described by } \\
\text { specific physical quantities (mass, } \\
\text { temperature, radius). Spherical shape is } \\
\text { justified in terms of gravity. Internal } \\
\text { structure and state of aggregation are } \\
\text { described in terms of variable density from } \\
\text { inner core to outer layers. }\end{array}$ & $\begin{array}{l}\text { The role of gravity as a central force in stars' } \\
\text { formation is correctly recognized. Stars' } \\
\text { functioning is justified in terms of nuclear } \\
\text { reactions and heat transfer mechanisms, } \\
\text { establishing a relationship between } \\
\text { surface temperature and inner processes. } \\
\text { Life/death stages of a star are related to the } \\
\text { star's initial mass }\end{array}$ \\
\hline
\end{tabular}

Level 4 Role of thermodynamics in stars' equilibrium is correctly recognized, but mechanism that relates balancing pressure forces to nuclear reactions is partially understood or unclear

Level 3 Unbalancing of gravitational S force is correctly recognized but no further mechanism to explain stars' equilibrium is provided
Stars are considered as celestial objects made mainly of $\mathrm{H}$ and $\mathrm{He}$ and described by specific physical quantities (mass, temperature, radius). Spherical shape is recognized but not justified in terms of gravity. Internal structure and state of aggregation are recognized but not clearly related to variable density from inner core to outer layers.
Gravity is recognized as attractive force but its role in star's birth is unclear. Stars' functioning is justified in terms of nuclear reactions and heat transfer mechanisms, establishing a relationship between surface temperature and inner processes. The role of mass in subsequent stages of the star's life, including death, is unclear 
updating of the upper anchor from facets is reported in the codebook as Supplemental Material 1 [35].

Functioning and evolution.-After the intervention, sixteen students used gravitational force in their reasoning to justify the process of stars' formation, though in a qualitative way.

For instance, S81 before the intervention held the false belief that a star was made by pieces of comets or planets that underwent collision. After the intervention, her reasoning showed some improvements:

I: "so how a star is formed?"

S81: "During a long time, gases that travel in the universe are attracted each other by gravity, which attracts everything in one point..."

I: "what is the form a star?"

S81: "they are spherical since there is a force that pulls everything towards the center..."

I: "and what is there, into one star's core?"

S81: "well... the star has a core, it is more compact, and nuclear reactions take place within it..."

Only two students were able, after the intervention, to exploit in more detail the role of gravity in SSE and to relate the temperature of the star to pressure through ideal gases state law (level 2). S95 showed before the intervention a correct view about the role of nuclear reactions for stars' functioning. After the intervention, he was able to add some meaningful details:

I: "so, why do you think a star has a spherical form?"

S95: "because at the center there are nuclear reactions... heavier particles are produced, and these particles attract other gases and smaller particles through gravity which acts in all directions... the latter remain trapped and rotate..."

I: "Can you better explain how nuclear reaction influence star's life?"

S95: "the radiation caused by nuclear reactions propagates as heat in stars' layers... the pressure inside the star is proportional to the temperature...."

This excerpt led to the introduction of a new level between level 2 and the upper anchor. The new level refers to the capability of relating surface temperature to pressure and nuclear processes inside the star. The construction of this new level from facets is reported in the codebook as Supplemental Material [35].

The above findings led to a new version of LP1 (LP2, Table V). The main differences with LP1 for the three dimensions are as follows:

hydrostatic equilibrium: two new levels (levels 3 and 4) were included to account for students' incomplete reasoning about forces in a star. The upper anchor was slightly rephrased to denote explicitly the forces that are involved in stars' equilibrium;

composition and aggregation state: a new level (level 3) was included to account for students' partial knowledge about stars' inner structure. The upper anchor was rephrased to account for students' justification of stars' shape in terms of gravity and knowledge about variable density.

functioning and evolution: a new level (level 3) was included to account for the students' partial knowledge about the relationships between surface temperature and inner processes. The upper anchor was correspondingly changed.

\section{Revision of TLS1}

Results of the study with sample 2 informed also the revision of TLS1. The resulting TLS2 included the following changes concerning the hydrostatic equilibrium dimension:

(i) the paper-and-pencil task on forces acting on a small element of mass is proposed before performing the measurement with Tracker of the rotational velocity of the Sun;

(ii) a new explanation of a star's equilibrium is proposed building on buoyancy and Archimedes' principle. In particular, we focused on the analogy between pressure forces acting on a body immersed in water and pressure forces due to nuclear reactions acting on a small element of mass.

In the composition and aggregation dimension, demonstration experiments using a spectrometer were introduced, the aim being to show how light spectra can be used to gain knowledge about the internal structure or processes of a not accessible source, as the star's core (Fig 11).

First, through spectral analysis of light emitted by incandescent lamps, students can infer that stars' emission of light is due to a thermal emission mechanism. Second, the measurement of the spectra of fluorescent and filament lamps is proposed to show a different physical process for producing light-stimulated emission. The difference between the absorption and emission spectrum is introduced and students are involved in a paper-and-pencil task to identify chemical absorption lines in the solar spectrum. In such a way, students may become aware of the existence in the outer layers of the stars of chemical elements that are different from $\mathrm{H}$ and $\mathrm{He}$.

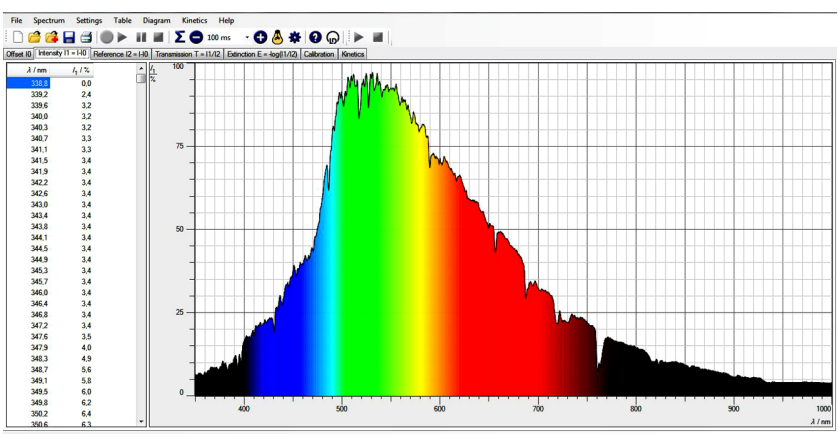

FIG. 11. Sun's spectrum measured through a spectrometer. The spectrum is not calibrated in intensity. 
The justification for the presence of $\mathrm{H}$ in stars' inner layers is discussed in the following activities concerning the functioning and evolution dimensions:

(i) a new paper-and-pencil task is proposed to estimate how much energy is released from burning a mass of $\mathrm{H}$, equivalent to that of the Sun and how long the Sun could live by emitting this amount of energy if only burning reactions involving $\mathrm{H}$ took place;

(ii) a new session is proposed to introduce the binding energy of chemical elements, from which one can determine the amount of energy produced in each nuclear reaction.

The new activities allowed for further justification of mechanisms underlying hydrostatic equilibrium and composition of the star. The time duration of the revised teaching intervention had to be increased up to $20 \mathrm{~h}$. More details can be found in Ref. [46] and in the Supplemental Material 2 [35].

\section{E. Design of the final LP}

In this section, we report on the results of the implementation of TLS2. Table VI reports the sample 3 students' distribution in the levels of LP2 before and after the instructional intervention. Preinstruction distribution was not significantly different to that of the 17-18 years old students of sample 1 and of sample 2 for all dimensions $\left(\chi^{2}=4.925, \quad \mathrm{df}=4, \quad p=0.295 ; \chi^{2}=6.564, \quad \mathrm{df}=6\right.$, $p=0.363 ; \chi^{2}=3.713, \mathrm{df}=2, p=0.156$, respectively). Analysis of interview responses shows significant improvements in students' understanding about SSE (marginal homogeneity test: $p<10^{-4}$ ).

Hydrostatic equilibrium.-After the intervention, twentyfive out 30 students could use Newton's law to explain stars' equilibrium or at least to justify why centripetal net force and gravitational force have different magnitudes. For instance, S121 was initially convinced that a star is not in equilibrium since there are gases inside of it that "burn" during all the star's lifetime. The same student after the intervention shifted his reasoning on forces.

TABLE VI. Sample 3 students' distribution across LP2 levels.

\begin{tabular}{|c|c|c|c|c|c|c|}
\hline \multirow[b]{2}{*}{ Level } & \multicolumn{2}{|c|}{$\begin{array}{l}\text { Hydrostatic } \\
\text { equilibrium }\end{array}$} & \multicolumn{2}{|c|}{$\begin{array}{l}\text { Composition and } \\
\text { aggregation state }\end{array}$} & \multicolumn{2}{|c|}{$\begin{array}{l}\text { Functioning } \\
\text { and evolution }\end{array}$} \\
\hline & Pre & Post & Pre & Post & Pre & Post \\
\hline Upper anchor & 0 & 5 & 0 & 5 & 0 & 4 \\
\hline Level 4 & 0 & 10 & $\ldots$ & $\ldots$ & $\ldots$ & $\ldots$ \\
\hline Level 3 & 0 & 15 & 0 & 6 & 0 & 6 \\
\hline Level 2 & 23 & 0 & 7 & 7 & 0 & 9 \\
\hline Level 1 & 7 & 0 & 15 & 10 & 12 & 8 \\
\hline Lower anchor & 0 & 0 & 8 & 2 & 18 & 3 \\
\hline Total & 30 & 30 & 30 & 30 & 30 & 30 \\
\hline
\end{tabular}

I: "what about the star's equilibrium?"

S121: "a star is in equilibrium since gravity is balanced by inner forces..."

I: "what do you mean by "inner" forces?"

S121: "centripetal acceleration is much smaller than gravitational acceleration... we made the calculation... so, there must some other force.... it is like in fluids... there is Archimedes' buoyant force..."

However, despite that he correctly identified the need for including inner forces that contribute to the star's equilibrium, S121 was not able to qualitatively justify in more detail the nature of such forces:

I: “... do you have any idea about the origin of these inner forces?"

S121: "uhm.. I don't know..."

I: "do you remember your answer before the activities?"

S121: ".. uhm.. yes.. I think that I was referring to something that is burning in the star..."

I: "what is this "something"?"

S121: "H and He..."

I: “... and how they contribute to equilibrium...?"

S121: “... I don't know... the star is in equilibrium... but, inside, the star is not in thermal equilibrium... (after a while).. ah I know! those forces depend on the processes that happen within a star..."

Five students showed a reasoning that aligns with the upper anchor. For instance, S113 before the intervention showed a typical misconception about circular motion (Gardner, 1984):

S113: "the star is in equilibrium because gravity equals centrifugal force" (Fig. 12)

After the intervention, she could add some kind of mechanism to justify stars' equilibrium (upper anchor):

S113: “...No, I was wrong, gravity is not balanced by centrifugal force"

I: “... Why?”

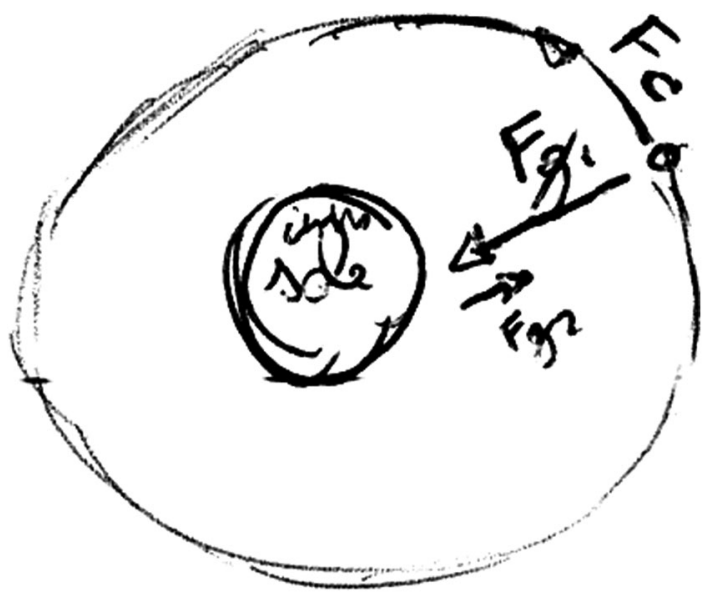

FIG. 12. Sample 3 student preinstruction sketch of forces acting on a small volume element of the Sun. Translation of Italian label: Sun. 


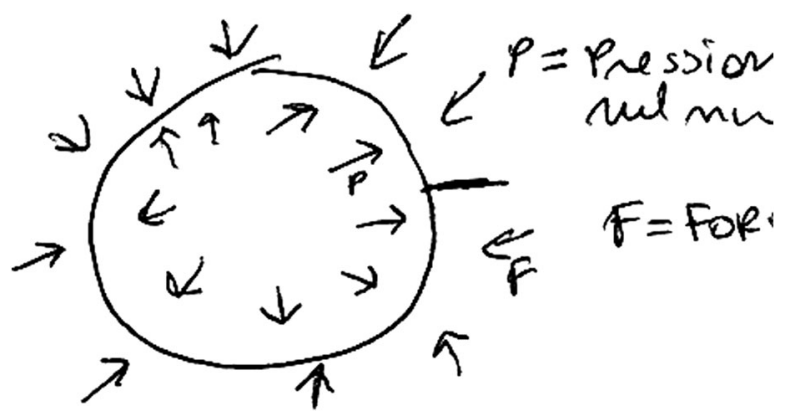

FIG. 13. Sample 3 student post-instruction sketch of forces acting on a small volume element of the Sun. Translation of Italian label: $P=$ Pressure in the core; $F=$ Force.

S113: “... I am not sure... but I think that the new force can only be related to nuclear reactions...."

I: "Why do you think so?"

S113: “... nuclear processes generate heat and energy that is transmitted somehow within the star ...it is this energy that balances gravity... I think..."

I: "Ok, but can you figure out some kind of mechanism? Think about what happens in perfect gases... You can make a drawing"

S113: “... uhm... yes...(she draws the sketch in Fig. 13) ... given high temperature, particles of a layer are moving faster and hit particles of other layers... and so they create upward pressure that balances gravity..."

By comparing the pre- and postinstruction interview, we note that the intermediate levels 2 and 3 captured the partial understanding of equilibrium in terms of unbalanced gravitational force first and then in terms of "buoyancy" forces that concur to star's stability. Such findings suggest that levels of LP2 adequately described students' progression in understanding this dimension of SSE.

Composition or aggregation.-The great majority of students (28 out of 30) were able to at least describe correctly chemical and physical composition of a star in terms of $\mathrm{H}$ and $\mathrm{He}$. Seven of them showed good knowledge of a star's inner layers, but they were not able to correctly justify changes in the density from the core to the outer shells. Five of them were able to justify correctly stellar composition, and they all referred to results obtained through spectral analysis of emitted light.

$\mathrm{S} 120$, for instance, in the precourse interview, claimed that stars were formed only by $\mathrm{H}$ and $\mathrm{He}$ because they are the most abundant element in the Universe. After the intervention, he answered,

I: "So, which elements are stars made the most?"

S120: "well... There are many... not only $\mathrm{H}$ and He..."

I: "Can you justify for example the solar chemical composition?"

S120: “... well I remember that we saw the solar spectrum and those black lines... There are a lot of elements in stars, produced by nuclear fusion reactions..." I: "what were those black lines?"
S120: "I think elements that are present in our atmosphere"

I: "is it possible to measure the solar spectrum disregarding the effect of our atmosphere?"

S120: "..yes! From outer space..."

I: "so what were those black lines?"

S120: ".. so they were elements in the outer layers of the Sun... I remember oxygen.. but also others...sodium, for instance...".

As a general trend for this dimension, we noted that the demonstration experiment with the spectrometer was important to advance students' knowledge from lower levels of LP2 towards higher levels and upper anchor. The students who did not refer to the spectrometer activity in their answers were not able to correctly justify the composition of a star, showing a knowledge limited to the presence of $\mathrm{H}, \mathrm{He}$; moreover, they only listed the names of subsequent layers inside a star, but they were not able to describe their density or their composition. For such reasons, we revised LP2 and added a further intermediate level 4 in which the role of chemical elements is acknowledged in influencing the spectrum of the light emitted by a star. Correspondingly, we modified the upper anchor to include a more sophisticated reasoning in which the presence of elements is correctly related to absorption lines superimposed to a blackbody radiation spectrum.

Functioning and evolution.-Twenty-seven out of 30 students were able at least to refer to nuclear reactions in the functioning of a star and to gravity in the stars' formation. S124 before the intervention answered that, inside the stars, chemical reactions take place. After the intervention, she considerably modified her answer:

I: "what is the main mechanism underlying stars' functioning?"

S124: "... uhm... the nuclear reactions that happen in its inner core... This is energy that atoms acquire through collisions..."

I: "what is it the main consequence of these reactions?"

S124: "...they produce heat and increase temperature...."

I: "what is the consequence of such increase?"

S124: "well... if temperature increases also pressure increases... the pressure pushes gravity... that's why a star is in equilibrium..."

I: “... yes... but are there other consequences of these reactions?"

S124: "well...they change the state of a star..."

I: "what do you mean by "state" of a star?"

S124: "well... a star changes its state when it changes its temperature... not only pressure changes... also the luminosity... when there are no more elements to transform the star change its brightness... hence, the brightness depends on the nuclear reactions that take place in the core of the star..."

I: "How is it possible?" 
TABLE VII. Final LP about SSE (LP3). Lower anchor to level 3 are as in LP1 (Table II) and LP2 (Table V).

\begin{tabular}{|c|c|c|c|}
\hline Level & Hydrostatic equilibrium & Composition and aggregation state & Functioning and evolution \\
\hline $\begin{array}{l}\text { Upper } \\
\text { Anchor }\end{array}$ & $\begin{array}{l}\text { Equilibrium of a star is } \\
\text { justified by balancing } \\
\text { gravitational force and } \\
\text { pressure forces, related to } \\
\text { nuclear reactions, on a } \\
\text { star's element of volume }\end{array}$ & $\begin{array}{l}\text { Stars are considered as celestial objects } \\
\text { described by specific physical quantities } \\
\text { (mass, temperature, radius) and made } \\
\text { mainly by H and He. Spectra are } \\
\text { recognized as the result of stars' } \\
\text { composition. The presence of elements } \\
\text { heavier than H and He is justified by } \\
\text { observed spectral absorption lines. } \\
\text { Spherical shape is justified in terms of } \\
\text { gravity. Internal structure and state of } \\
\text { aggregation are described in terms of } \\
\text { variable density from inner core to outer } \\
\text { layers. }\end{array}$ & $\begin{array}{l}\text { Gravity is recognized as attractive force that } \\
\text { determines the spherical shape of a star } \\
\text { being a force with central symmetry. } \\
\text { Stars' functioning is described as radiation } \\
\text { emission and justified in terms of nuclear } \\
\text { reactions and heat transfer mechanisms, } \\
\text { establishing a relationship between } \\
\text { surface temperature and inner processes. } \\
\text { Nuclear reactions are related to stars' } \\
\text { evolution and production of elements until } \\
\text { iron, after which fusion is no longer } \\
\text { favorable from energy viewpoint. Life/ } \\
\text { death stages of a star are qualitatively } \\
\text { justified in terms of initial mass }\end{array}$ \\
\hline Level 4 & $\begin{array}{l}\text { Role of thermodynamics in } \\
\text { stars' equilibrium is } \\
\text { correctly recognized, but } \\
\text { mechanism that relates } \\
\text { balancing pressure forces } \\
\text { to nuclear reactions is } \\
\text { unclear }\end{array}$ & $\begin{array}{l}\text { Stars are considered as celestial objects } \\
\text { described by specific physical quantities } \\
\text { (mass, temperature, radius) and made } \\
\text { mainly by H and He. Spectra are } \\
\text { recognized as the result of stars' } \\
\text { composition. Spherical shape is } \\
\text { recognized but not justified in terms of } \\
\text { gravity. Internal structure and state of } \\
\text { aggregation are recognized but not related } \\
\text { to variable density from inner core to outer } \\
\text { layers. }\end{array}$ & $\begin{array}{l}\text { Gravity is recognized as attractive force but } \\
\text { its role in star's birth is partially described. } \\
\text { Stars' functioning is described as radiation } \\
\text { emission and justified in terms of nuclear } \\
\text { reactions and heat transfer mechanisms, } \\
\text { establishing a relationship between } \\
\text { surface temperature and inner processes. } \\
\text { Nuclear reactions are related to stars' } \\
\text { evolution and production of elements until } \\
\text { iron. The role of mass in subsequent } \\
\text { stages of the star's life, including death, is } \\
\text { unclear }\end{array}$ \\
\hline
\end{tabular}

S124: "well... Electrons jump between atomic levels since they are excited by the heat transmitted within the star... the more the temperature the more the jump..."

S124 therefore seems to have grasped the relationships between nuclear reactions, equilibrium, light emission, and composition but the underlying mechanisms seem yet not clear. It is worth noting that only four students claimed qualitatively that the final stages of a star depend on the initial mass.

For instance, S117 before the module answered that a star, after having consumed all the $\mathrm{H}$ and $\mathrm{He}$, would become a black hole or a white dwarf since no more nuclear reaction could take place. After the intervention, he slightly changed his answer:

I: "How would you describe the final stages of a star?"

S117: "well... nuclear reactions do not happen anymore..."

I: "can you explain why?"

S117: "...well there's no more mass .."

I: "what do you mean?"

S117: "well... nuclear reactions depend on the mass..."

I: "ok... can you explain in more detail? What happens

to the star when there's no more mass?"

S117: “...well, actually its death depends on the previous mass...we have seen that if only chemical reactions took place, the Sun would not have lasted until our days..."
I: "yes, and so?"

S117: "... there must be something that "tells" the core to stop with the reactions... also light emitted by a star depends on temperature and nuclear reactions... they change their light in the final stages... so I think that this something is the mass..."

This excerpt led us to revise LP2 also in the functioning and evolution dimension by adding a further level (level 4) to describe students' reasoning about the relationship between light emission and inner nuclear reactions and the evidence that after the Fe element, nuclear reactions are no longer energetically favorable. Accordingly, we also slightly changed the formulation of upper anchor. The final obtained LP3 is reported in Table VII. A complete description of the levels, including hypothesized patterns of students' reasoning across progress variables are reported in Appendix B.

\section{DISCUSSION}

The aim of this study was to investigate the qualitative distinctions between increasingly sophisticated knowledge and reasoning about the SSE big idea in response to curriculum and specially designed teaching activities. SSE was considered as suitable to develop a LP in its own right first because it is an explanatory model at the 
basis of accepted theories about the Universe. Second, it satisfies the following three criteria proposed in Ref. [8] for choosing big ideas: (i) to represent relevant ways of knowing and understanding the universe; (ii) to describe explanatory models that can be initially taught starting from students' observations of the world; (iii) to explain multiple, unified astronomical phenomena. Finally, SSE is a generative educational context that harnesses several core ideas in basic and advanced physics and chemistry, as equilibrium, energy, atoms, nature of matter, and chemical reactions. Building on previous studies and on curriculum teaching about SSE, we chose to describe the knowledge base about SSE along three interrelated dimensions: hydrostatic equilibrium, composition and aggregation state, functioning and evolution. Given the dearth of research we had to develop empirically the levels of the hypothetical LP about SSE starting from students' ideas. The development process was iterated through three cycles of hypothesis, implementation, and refinement with the aim of fine tuning students' understanding with the proposed levels for the three dimensions. The use of the facets approach allowed us to construct a fine-grained LP. Such a methodological choice allowed us to better adapt the levels to students' reasoning and knowledge, rather than force their location into predetermined levels. To empirically support such levels, we designed a teaching module that could reflect students' thinking about the addressed concepts [48] and support students' progress through the levels of the LP [49]. The module itself was revised after each implementation. Data collected during the implementations enabled us to improve mapping of students' reasoning onto LP levels and provided insights about the difficulty students experience explaining SSE [16]. In particular, the findings of this study support that the three chosen dimensions form a meaningful knowledge base for interpreting students' reasoning about SSE along different high school levels. Our choice was guided by the rationale that the three dimensions (i) could explain a variety of phenomena concerning SSE; (ii) are representative of the concepts addressed in the teaching of SSE in high school curricula; and (iii) encompass students' relevant ideas. Other dimensions could have been chosen according to a different choice of school level, resulting in a different LP. For instance, a possible different dimension for a LP about SSE that would start from the middle school level could include topics such as the difference between stars and planets, the difference between the Sun and stars, and the distance between stars and Earth [9]. On the other hand, our choice fits in a better way advanced teaching about SSE, as typical ASTRO 101 courses [32] or graduate astrophysics courses. Our results confirm and extend previous studies in astronomy education research. Concerning the hydrostatic equilibrium dimension, ideas of students about the role of gravity in stars' formation and stability confirm findings in Ref. [12], where they found that at lower levels of their LP about the
Solar System, students did not use gravity to justify the process of matter aggregation in the formation of the planets and the Sun. To explain such a result, they suggested a difficulty of students in recognizing that gases have masses. While this may be a challenge for middle school students, what was most difficult for the students in our samples (high school students, 13-18 years old) was not to recognize the role of gravity in the formation of stars, but to justify their spherical shape, likely because they had not fully understood that gravity is a central force. Similarly, our findings show that high school students did not find it challenging to identify gravitational force as relevant for the equilibrium of a star. Rather, students had difficulty identifying the balancing force, likely because (i) they held the misconception that a circular motion implies the existence of a centrifugal force; (ii) the concept of pressure forces is usually applied only in the context of the statics of liquids. In the composition or aggregation dimension, our findings confirm previous results [33] for which students have no particular difficulty identifying $\mathrm{H}$ and $\mathrm{He}$ as the main elements of stars. However, our results show that such knowledge likely does not go beyond a rote learning since only a minority of students, even after being exposed to specific teaching activities with the spectrometer, was able to at least indicate the presence of other elements in stars. Such difficulty appears to be related to the well-known lack of knowledge about the nature of chemical elements and to a naïve view of matter [50]. Difficulty with stars' composition was likely due also to a scarce knowledge of stars' functioning, in particular of the role of nuclear reactions. A first result was that, although all students in samples 2 and 3 had been taught about the HR diagram and hence knew the relationships between brightness and temperature of a star during its evolution, none of them was able to justify or interpret evolution of a star in terms of nuclear reactions. Such evidence can be primarily related to the confusion between nuclear and chemical reactions [16,33]. The idea that hydrogen and other gases are burnt within a star was addressed showing the calculation of how long the Sun would have lasted if reactions in stars were burning reactions and not nuclear reactions. However, some difficulty still emerged when students had to deal with how nuclear reactions produce chemical elements and why production ends up with the $\mathrm{Fe}$ element. Thus, to fully understand the mechanisms underlying stellar evolution, instruction may need to go beyond a superficial knowledge of stars' evolution in terms of a taxonomy of stages in the HR diagram and address in a clearer way atomic behavior and structure of matter.

A second aspect that is worth discussing concerns curriculum teaching about SSE. Our findings related to LP1 support that earth sciences and physics high school teaching provides students with a limited ability in explaining SSE according to a scientific view. For instance, none of 77 interviewee students of sample 1 was able to justify 
with a correct explanation how gravity is balanced within stars or how final stages of a star's life depend on the initial mass and the nuclear fusion processes. Such results are similar to those reported in Refs. [10,30]. Moreover, we found that students that were exposed to a typical curriculum intervention had a scarce knowledge of many relevant concepts related to SSE. Such a result is particularly significant concerning the hydrostatic equilibrium and the composition or aggregation dimensions, whose levels are informed by knowledge of Newton's second law, statics of fluids, and chemical composition; topics that are usually taught at the high school level. Such data, in agreement with previous studies [14], show that most high school students do not hold a sophisticated knowledge of physics and chemistry and find it difficult to apply previously learned laws to a nonusual context, as stars. To this concern, our findings extend those of previous studies $[15,33]$ showing that students' knowledge about SSE is often fragmented and inconsistent. For instance, the consuming model of nuclear reactions can coexist with an equilibrium model in which the loss of mass and energy does not have implications on equilibrium, since mechanical and thermodynamics phenomena are treated separately. Similarly, although forces acting on stars' volume element are correctly identified, a model of a star as an unstable object could still be acceptable to students, since changes in the mass as a star evolves are not correctly interpreted as result of changes in the nuclear fusion reactions.

\section{CONCLUSIONS AND IMPLICATIONS}

This study can contribute broadly to the LP research field as an example of the interplay between the investigation into students' reasoning and the design of research-based teaching modules. To our knowledge, ours is the first LP in astronomy that is developed and revised in strict correspondence to a teaching module. Such a research development is rare also in the broad field of science education since few studies have so far described subsequent cycles of revisions after implementation of teaching activities [51]. Thus, our study may be helpful for researchers in the field who aim at developing evidence-based LPs. To this concern, we focus LP scholars' attention on the implications of our study.

First, by showing changes in students' reasoning before and after the teaching activities, we can model how progressions through the levels of the LP depend on the designed instruction. In particular, findings support the evidence that, in the case of stars' structure, spectrometer experiments allowed the students to link their basic knowledge about nuclear reactions, to a new knowledge that involves consequences of nuclear reactions, as surface temperature of a star and the production of chemical elements. Having deepened the knowledge about nuclear reactions and their consequences, students involved in our instructional activities were allowed to grasp also a sounder understanding of why mass is fundamental in determining the evolution of a star. In such a way, the designed activities were effective in helping students reach intermediate levels of the LP, between the lower and upper anchor.

A second implication for research in LPs is that suitably designed instructional activities may be useful to link different dimensions of a given big idea. Data collected with sample 1 show that the Tracker activity on the estimation of the Sun's rotational speed was not sufficient to help students understand equilibrium in stars since it showed only that centripetal acceleration does not have the same magnitude of gravitation acceleration, giving no further clues about how gravity is actually balanced. For this reason, the majority of students of sample 2, while leaving a naïve not-equilibrium model, still held an incorrect view of the balance of gravitational force. It was only after the introduction of the analogy between the equilibrium in stars and buoyancy in liquids that students, building on their own knowledge from hydrostatics, began to introduce in their reasoning pressure forces. As one of the reported interview excerpts shows, to have understood the role of pressure forces in the equilibrium of a star likely helped students to link, at least qualitatively, about equilibrium, composition, and functioning of a star, thus linking gravity, energy transportation, and nuclear reactions in a star.

To this same concern, our results support the conclusion that the capability to reason about SSE improves if students are exposed to teaching activities that address not only basic astronomy notions about stars, but also physics laws that students already have been taught about in high school when studying mechanics, hydrostatics, and thermodynamics. The fact that such laws are already featured in the curriculum supports also the usability of our LP in current teaching practice, without the need to spend a great amount of teaching time to address out-of-curriculum topics.

Consequently, and as a more general implication for physics education, our study shows that SSE can be a fruitful context to develop a more coherent and profound knowledge of core ideas in physics. Further instructional activities using SSE as context, can be extended to support students' learning of advanced physics topics as matterradiation interaction and quantum mechanics. The teaching module that we developed to iteratively validate the hypothesized LP may be a useful step towards effective teaching strategies that support students in this effort. Moreover, given the interdisciplinary nature of the identified dimensions of the SSE big idea, further research is required to investigate whether the proposed instructional activities may help students progress towards more sophisticated understanding also of other big ideas in science, such as force and motion, nature of matter, and energy.

\section{LIMITATIONS OF THE STUDY}

Limitations of this study include the lack of data about how single students develop their understanding about SSE 
along the five years of the Italian high school curriculum. Such a longitudinal study would be the ideal route to validate hypothetical LPs [19], but due to local constraints (e.g., frequent change of teachers throughout the years), we found problems in implementing such an approach. We therefore resorted to a more pragmatic approach and followed single students as they moved along the levels of the subsequent LPs at a smaller time scale $(20 \mathrm{~h})$. In such a way, we were able to specify in more detail the learning goals that describe students' understanding at a given level of the LPs. As we developed also specific teaching activities to support students in progressing from one level to another, our study may envisage specific learning trajectories that students may follow when learning about SSE [52]. However, as usual in the LP research, our study describes only a tentative progression that students may follow as they develop views and models about stars that are increasingly more sophisticated. Clearly, students can follow alternative pathways along the three dimensions, in response to specific teaching interventions. For instance, students could reason about stellar evolution by starting from a comparison of the mass of a star with respect to that of the Sun, or taking into account a star's brightness. Such an alternative pathway along the LP levels would reflect in different objectives and activities of the proposed teaching module.

A further limitation is that the sample, consisting of high school students, did not include predictably lower anchored students (e.g., middle school students) and upper anchored students (e.g., undergraduate astrophysics students). While the aim of this study was mainly to make first steps in developing a LP about SSE and to construct its levels in strict connection to specific teaching activities so that the LP could be more responsive to students' progression in thinking, the inclusion of a sample spanning over a broader age range would have likely strengthened the empirical evidence for the levels of the three dimensions.

Two final limitations should be reported, namely, the small number of items in the interview and the small size of samples 2 and 3. While the interview protocol provided us access to a rich picture of students' initial knowledge about SSE, more specific questions could have been useful to clarify in more detail students' reasoning at intermediate levels. Concerning the small number of students involved in the validation of LP1 and LP2, we note that we needed an in-depth analysis of students' reasonings to improve our description of levels near the upper anchor. While both limitations do not affect findings of the current study, it is likely that LP3 levels would need more revisions using an improved research tool and larger samples.

\section{ACKNOWLEDGMENTS}

Grateful thanks go to the teachers and pupils of the schools involved: Emilio Segrè High School in S. Cipriano d'Aversa (Italy); Publio Virgilio Marone in Meta di Sorrento (Italy); Giovan Battista Vico High School in Naples (Italy);
Filippo Silvestri High School in Naples (Italy). This research was funded by the Ministero dell'Istruzione, dell'Università e della Ricerca in the framework of the Scientific Degrees National Project [53].

\section{APPENDIX A: COMPLETE INTERVIEW PROTOCOL}

Part I-Introduction

The interviewer begins with the following introduction:

"During the following three quarters of hour, we will be talking about several aspects that are of general concern about the topic of stars, that you have recently studied in your science and physics class [for sample 2 and 3, in the activities we have carried out at the department of physics). I am not concerned with how much you have studied, you will not get any grade from me or from your teacher. I am interested in how you think about stars, their evolution and functioning. To standardize what we will talk about, I will be asking first a general question, and then we will go into details, depending on your answer. Are there any questions before we begin?".

Then, the interviewer informs the participant of the fact that the interview will be tape recorded.

Part II-List of standard probe questions:

(1) What is a star?

(2) What is the composition of a star?

(3) Draw the shape and internal structure of a star. Briefly explain your drawing.

(4) Do you think that a star is a system in equilibrium?

(5) How does a star form?

(6) What is the main mechanism underlying stars' functioning?

(7) How would you describe the final stages of the life of a star?

Part III-General suggestions for conducting the interview

(a) If the response to a given probe question is incomplete, ambiguous, contradictory, or simply unclear, the interviewer can ask to further elaborate by asking such questions as:

Can you give me some justification for...

What do you mean by... .

Why do you think that...

Do you have any idea about why...

Can you explain in more detail...

Can you make a drawing or a sketch about...?

Can you explain your drawing?

(b) If the participant replies with a standardized answer or formula, the interviewer may reformulate the answer of the student and ask for further justification:

So, you think that..... Why do you think that?

Yes, you claim that..., but can you explain it with different words?

You said that... can you give me some justification for...?

(c) If the participant is struggling to find words or shows difficulty in clarifying her or his thinking, the interviewer 
may also go into detail depending on the specific topic of the question, asking for more clarifications. For instance, the interviewer may refer to specific activities done during the module in order to elicit students' reasoning. Or, the interviewer may specifically refer to some aspects that are not clear or that are relevant for the discussion:

Do you remember what you have done about...?

What can you conclude from your data about....?

You have seen that.... What if ....?

Part IV-Question by question specific suggestions for conducting the interview (see also text for further suggestions)

Question 1: if they answer referring to celestial objects, probe further asking:- - what kind of celestial objects? Are they similar to planets? If yes/no, ask for explanation. If they talk about qualities as being hot/cold or being small/ big, investigate further asking:- why do you think that they are hot/cold? - what is their temperature?-do their size change? If rotation comes is ask for justification:-why does a star must rotate?-have stars angular momentum?

Question 2: if they do not go further than saying that a star is formed by $\mathrm{H}$ and $\mathrm{He}$, then continue to find out what is their view about the fundamental structure of matter or ask them to describe in more detail the distribution or aggregation state of the elements in a star. Ask further:what evidence do you have to justify solar composition? If light spectra are recalled, ask for clarification:-how do we measure such spectra?-what are the most important features of a spectrum? If the student still has difficulties say that it is possible to make a drawing. When s/he has produced the drawing, then ask to explain it and build on the visual representation to investigate further the student' ideas. If they represent $\mathrm{H}$ and $\mathrm{He}$ as particles, ask for instance:-what are these dots?-what are these balls? If the particles are drawn very close or a specific pattern emerges, ask: how is it that they are so close?-why are they arranged in this way?-how have they come to such configuration?-is there anything different about the particles at the center or at the edge of a star? If they get into a continuous-based representation, then ask:-is it like a gas? Is it like a liquid? If plasma comes into the discussion as aggregation state, then ask:- how do you imagine it?- to what extent is it different from other states of matter?

Question 3: if they draw a circle with spikes ask:-what are these spikes? - how are they formed?-what do they represent? - can you be more specific about the shape of the edge of the star? If a circle is drawn ask for reasons of such a shape. Investigate further by asking:- what is the physical cause for such a shape? - can you make an example in a different context? Return to this question during discussion in question 6.

Question 4: if yes/no, ask for further clarification and for a drawing:- can you give me some justification for such equilibrium? - can you make a drawing? In the explanation, if centrifugal force comes in, then ask:-what causes such force?-can you specify the reference system you have adopted? If "reaction" or "inner reaction" comes in, then ask:-what is the origin of such reaction?-what do you mean by "inner"? If energy-force balance comes in, then ask:- how can energy be compared to a force?-where does such energy come from? In the drawing, if arrows are drawn in a body-free diagram then ask:-what does this arrow represent? —can you label each arrow? — can you compare the magnitudes of these forces? - are there any other forces that are involved? If curved arrows appear, ask for clarification:-what is such curved arrow? - what force does is represent?-is it related to rotation?

Question 5: if s/he answers that a star is formed from particles, dust, detritus, pieces of other celestial objects, investigate further by asking:- - how such dust/particles are pushed together?-is there any physical agent that causes such aggregation? If they get to gravity but do not say anything about it being a central force, then ask:-what is the direction of such force? — can you make an example? If necessary, investigate further by asking to make a drawing: if particles are put in a circular shape, then ask:-can you relate such shape to a specific physical cause? If particles are in no specific order, then ask:- - what evidence do you have for such structure?-will the particles maintain their initial configuration?-how is it possible to change it?

Question 6: if s/he answers that reactions happen in a star, probe further asking:- can you tell me more about such reactions? - can you make an example?-what are the consequences of such reactions?-do they transform elements one into another? If they refer to chemical reactions, ask:-what evidence do you have that chemical reactions happen in a star? - can you make an example of chemical reaction that happens in the Sun?- to what extent are such reactions important for the life of a star? If they then recall nuclear reactions ask:-ok, but tell me more about such reactions: are they similar or different from chemical ones? If yes/no, ask for further explanations and comparisons.

Question 7: if answer concerns black hole or explosion then ask:-what is your idea about a black hole?-will also our Sun explode?- - how is that a star can explode? Investigate further by asking:- can you say what happens to its temperature/pressure/volume at these final stages?what happen to the nuclear reactions?-do they continue, or they stop?-how is it possible that we find elements heavy metals in the universe? If mass does not come in as the main factor affecting a star' evolution, then ask:-can we predict what will happen to a star?-how can scientists know what will happen to our Sun?

\section{APPENDIX B: COMPLETE DESCRIPTION OF THE FINAL SSE LP3 LEVELS}

\section{Hydrostatic equilibrium dimension}

Lower anchor: students at this level consider a star as a system not in equilibrium because it evolves with time or because they confuse mechanical and thermal equilibrium. 
Level 1: students at this level are aware that stars are in equilibrium although no mechanism is provided. They may be aware of stellar observations but do not link their reasoning to physical quantities.

Level 2. Students at this level are aware that gravitational force would make a star collapse, but they incorrectly balance gravitational force with other forces (e.g., centrifugal force). Further, they may inaccurately balance gravitational force with energy produced in some way within the star. They do not consider any forces related to the inner processes of the star that could contrast gravity.

Level 3. Students at this level correctly apply Newton's second law obtaining that the centripetal acceleration of a small volume element has a magnitude that is lesser than expected if gravity would be the only force acting on it. However, they are not able to identify the other forces at play in the star's stability.

Level 4. As level 3, students at this level are aware of the role of both the gravity and thermonuclear processes that happen within the star. However, they are also able to use hydrostatic reasoning to justify star's stability, but they are not able to relate forces that balance gravity to nuclear reactions.

Upper anchor. Students at this level are able to balance gravitational force with radiative pressure forces. They are also able to explain that pressure forces arise from nuclear reactions. In particular, they are able to consistently use thermodynamics reasoning to relate increase in star's temperature with increase in pressure.

\section{Composition and aggregation state dimension}

Lower anchor: students at this level think that stars are made of solid particles, as dust, or pieces of planets, or that stars are the result of collisions of other celestial bodies. They can be aware that $\mathrm{H}$ and $\mathrm{He}$ are components of the stars but do not consider these gases as the main elements responsible for a star's composition. Furthermore, they may describe stars' shape as irregular due to the distribution of materials and gases

Level 1: students at this level are able to describe stars as object made of gases, mainly $\mathrm{H}$ and He. However, they are not able to identify physical quantities that describe stars. They consider stars as having a spherical shape, but they are not able to describe internal structure and to explain the state of aggregation of matter inside the star.

Level 2: at this level, students start to consider stars as celestial as rotating objects made of $\mathrm{H}$ and $\mathrm{He}$. They begin to describe stars as objects described by physical quantities such as mass, temperature, volume, and luminosity. In addition, they are able identify the spherical shape of stars and a simple inner structure made of a nucleus and an external crown, but they are not able to justify these properties. However, they are not able to describe correctly the state of aggregation of matter inside the nucleus of the star

Level 3: students at this level are able to describe stars as rotating celestial objects made mainly of $\mathrm{H}$ and $\mathrm{He}$ with a certain mass, temperature, radius, and luminosity. They are able to model stars as spherical objects, but they are not able to justify the shape in terms of gravity. They begin to recognize that a star has an internal structure and they are able to describe the state of aggregation of matter inside the star. However, they are not able (or at best only partially) to relate internal structure and state of aggregation with a density that varies from the center to the external layers.

Level 4: In addition to reasoning developed at level 3, students at this level are able to relate the spectrum plot of the light emitted by a star to its chemical composition $r$, but they are not able to give a physical mechanism for such process.

Upper anchor: students at this level are able to justify a stars' spherical shape in terms of gravity and to describe stars' internal structure and state of aggregation in terms of density that varies from the center to the external layers. Moreover, they are also able to justify, using simple atomic models, the absorption lines in stars' spectra and to identify from such lines the elements that are produced within stars and that can be found in a star's atmosphere.

Functioning and evolution dimension

Lower anchor: Students at this level do not recognize the role of gravitational force in the stars' formation process. Although they recognize that nuclear reactions play an important role in a stars' functioning, they often confuse nuclear fusion with chemical reactions. At this level, students show many misconceptions about a stars' final stages, as, e.g., stars always explode or become black holes.

Level 1: Students at this level start to explain stars' formation as a generic attraction between dust and particles of a nebula. They are able to describe the role of nuclear reactions in the stars' functioning, but they are not able to justify how nuclear reactions influence stars' composition and stability. In addition, they do not consider that the mass has some role in the final stages of a star, so that misconceptions about a stars' death, at this level, do not vanish.

Level 2: At this level, students are aware that gravitational force is an attractive force, but they are not able to explain how gravity leads to a spherical form for stars. Students are able to explain stars' functioning in terms of nuclear reaction and to justify heat transfer mechanisms within a star. However, they are not able to justify a star's evolution and final stages in terms of initial mass.

Level 3: In addition to reasoning developed at level 2, students at this level are able to build on heat transfer mechanisms inside stars to establish a relationship between surface temperature and inner processes. The role of initial mass in a stars' evolution and death remains unclear.

Level 4: At this level, students justify a star's formation on the basis of gravitational force that attracts gases toward the center of the star. In addition, students are able to relate nuclear reactions to emission of radiation and to establish a relationship between the surface temperature and the radiation emitted. Students at this level are also able to read the energy binding plots and begin to explain the 
production of increasingly heavy elements using nuclear reactions until the production of Fe. However, they are not aware of the role of initial mass in processes of stars' functioning and the stage of star's evolution

Upper anchor: Students at this level are able to consistently use central symmetry of gravitational force to justify a stars' spherical shape. In addition to level 4, they are also able to justify from nuclei potential energy viewpoint why nuclear reactions are not favored after the creation of iron. Using their knowledge about nuclear reactions, students are able to justify how mass influences a star's evolution and death.
[1] T. Corcoran, F. A. Mosher, and A. Rogat, Learning Progressions in Science: An Evidence-Based Approach to Reform. (Consortium for Policy Research in Education, Philadelphia, 2009).

[2] J. S. Krajcik, L. M. Sutherland, K. Drago, and J. Merritt, The promise and value of learning progression research, in Making it Tangible: Learning Outcomes in Science Education, edited by S. Bernholt, K. Neumann, and P. Nentwig (Waxmann, Münster, 2012), pp. 261-284.

[3] R. Duschl, S. Maeng, and A. Sezen, Learning progressions and teaching sequences: A review and analysis, Stud. Sci. Educ. 47, 123 (2011).

[4] W. Breslyn, J. R. McGinnis, R. C. McDonald, and E. Hestness, Developing a learning progression for sea level rise, a major impact of climate change, J. Res. Sci. Teach. 53, 1471 (2016).

[5] T. Willard and J.E. Roseman, Progression of Understanding of the Reasons for Seasons (Knowledge Sharing Institute of the Center for Curriculum Materials in Science, Washington, DC, 2007).

[6] C. Sneider, V. Bar, and C. Cavanagh, Learning about seasons: A guide for teachers and curriculum developers, Astron. Educ. Rev. 10, 010103 (2011).

[7] J.D. Plummer and L. Maynard, Building a learning progression for celestial motion: An exploration of students' reasoning about the seasons, J. Res. Sci. Teach. 51, 902 (2014).

[8] J. D. Plummer, Challenges in developing, and validating an astronomy learning progression, in Learning Progressions in Science: Current Challenges, and Future Directions, edited by A. Alonzo and A. W. Gotwals (Sense Publishers, Rotterdam, 2012), pp. 77-100.

[9] J. D. Plummer, A Cross-age study of children's knowledge of apparent celestial motion, Int. J. Sci. Educ. 31, 1571 (2009).

[10] J.D. Plummer and J.S. Krajcik, Building a learning progression for celestial motion: Elementary levels from an earth-based perspective, J. Res. Sci. Teach. 47, 768 (2010).

[11] I. Testa, S. Galano, S. Leccia, and E. Puddu, Development and validation of a learning progression for change of seasons, solar and lunar eclipses, and moon phases, Phys. Rev. ST Phys. Educ. Res. 11, 020102 (2015).

[12] J. D. Plummer, C. Palma, A. Flarend, K. A. Rubin, Y. S. Ong, B. Botzer, S. McDonald, and T. Furman, Development of a learning progression for the formation of the Solar System, Int. J. Sci. Educ. 37, 1381 (2015).
[13] A. Lelliott and M. Rollnick, Big Ideas: A review of astronomy education research 1974-2008, Int. J. Sci. Educ. 32, 1771 (2010).

[14] A. G. Riess, L. M. Macri, S. L. Homann, D. Scolnic, S. Casertano, A. V. Filippenko, B. Tucker, M. J. Reid, D. O. Jones, J. M. Silverman, R. Chornock, P. Challis, W. Yuan, P. J. Brown, and R. J. Foley, A $2.4 \%$ determination of the local value of the Hubble constant, Astron. J. 826, 56 (2016).

[15] L. Agan, Stellar ideas: Exploring students' understanding of stars, Astron. Educ. Rev. 3, 77 (2004).

[16] J. M. Bailey, E. E. Prather, B. Johnson, and T. F. Slater, College students' pre-instructional ideas about stars and star formation, Astron. Educ. Rev. 8, 010110 (2009).

[17] M. Finegold and D. Pundak, Students' conceptual frameworks in astronomy, Aust. Sci. Teachers J. 36, 76 (1990).

[18] J. G. Sharp, Children's astronomical beliefs: A preliminary study of Year 6 children in south-west England, Int. J. Sci. Educ. 18, 685 (1996).

[19] Taking Science to School: Learning and Teaching Science in Grades $K-8$, edited by R. A. Duschl, H. A. Schweingruber, and A. Shouse (National Academy Press, Washington, DC, 2007).

[20] S. Y. Stevens, C. Delgado, and J. S. Krajcik, Developing a hypothetical multi-dimensional learning progression for the nature of matter, J. Res. Sci. Teach. 47, 687 (2010).

[21] L. Mohan and J. Plummer, Exploring challenges to defining learning progressions, in Learning Progressions in Science: Current Challenges and Future Directions, edited by A. C. Alonzo and A. W. Gotwals (Sense, Rotterdam, 2012), pp. 139-150.

[22] R. G. Duncan and C. E. Hmelo-Silver, Learning progressions: Aligning curriculum, instruction, and assessment, J. Res. Sci. Teach. 46, 606 (2009).

[23] C. L. Smith, M. Wiser, C. W. Anderson, and J. Krajcik, Implications of research on children's learning for standards and assessment: A proposed learning progression for matter and the atomic-molecular theory, Measurement: Inter. Res. and Persp. 4, 1 (2006).

[24] M. R. Wilson and M.W. Bertenthal, Systems for State Science Assessment (National Academies Press, Washington, DC, 2006).

[25] S. Carey, Conceptual Change in Childhood (MIT Press, Cambridge, MA, 1985).

[26] R. Driver, Making Sense of Secondary Science: Research Into Children's Ideas (Routledge, New York, 1994). 
[27] S. Vosniadou, On the Nature of Naïve Physics, in Reconsidering Conceptual Change: Issues in Theory and Practice, edited by M. Limòn and L. Mason (Springer, Netherlands, 2002).

[28] L. Mohan, J. Chen, and C. W. Anderson, Developing a multi-year learning progression for carbon cycling in socio-ecological systems. J. Res. Sci. Teach. 46, 675 (2009).

[29] K. L. Gunckel, L. Mohan, B. A. Covitt, and C. W. Anderson, In Learning progressions in science: Current challenges and future directions, edited by A. C. Alonzo and A.W. Gotwals (Sense, Rotterdam, 2012), pp. 39-75.

[30] Proceedings of the Learning Progressions in Science (LeaPS) Conference, Iowa City, IA, 2009, edited by A. Alonzo and A. Gotwals, available from http://education .msu.edu/projects/leaps/proceedings/Default.html.

[31] A. Collins, D. Joseph, and K. Bielaczyc, Design research: Theoretical and methodological issues, J. Learn. Sci. 13, 15 (2004).

[32] J. M. Bailey, B. Johnson, E. E. Prather, and T. F. Slater, Development and validation of the star properties concept inventory, Int. J. Sci. Educ. 34, 2257 (2012).

[33] J. M. Bailey, Development of a concept inventory to assess students' understanding and reasoning difficulties about the properties and formation of stars, Ph.D. thesis, The University of Arizona, Tucson, AZ, 2006.

[34] E. L. Palmieri and M. Parotto, Il Globo Terrestre e la sua evoluzione (The Terrestrial Globe and its Evolution) (Zanichelli, Bologna, 2014).

[35] See Supplemental Material at http://link.aps.org/ supplemental/10.1103/PhysRevPhysEducRes.14.010143 for 1) Codebook for the mapping of emerging facets into levels of SSE LP 2) Teachers' notes and students' worksheets of the module about SSE.

[36] J. Minstrell, Facets of students' knowledge, and relevant instruction, in Research in Physics Learning: Theoretical Issues and Empirical Studies, edited by R. Duit, F. Goldberg, and H. Niedderer (IPN, Kiel, 1992), pp. 110-128.

[37] I. Galili and A. Hazan, Learners' knowledge in optics: interpretation, structure and analysis, Int. J. Sci. Educ. 22, 57 (2000).

[38] A. H. DeBarger, C. Ayala, J. Minstrell, P. Kraus, and T. Stanford, Facet-based Progressions of Student Understanding in Chemistry (SRI International, Menlo Park, CA, 2009).
[39] J. Corbin and A. Strauss, Basics of Qualitative Research. Techniques and Procedures for Developing Grounded Theory (Sage Publications, London, 2015).

[40] X. Sun and Z. Yang, Generalized McNemar's test for homogeneity of the marginal distributions, Statistics and Data Analysis (SAS Global Forum, San Antonio, TX, 2008).

[41] P. Gardner, Circular motion: Some post-instructional alternative frameworks, Res. Sci. Educ. 14, 136 (1984).

[42] R. Gunstone, Circular motion: Some pre-instruction alternative frameworks, Res. Sci. Educ. 14, 125 (1984).

[43] C. Singh, Centripetal acceleration: Often forgotten or misinterpreted, Phys. Educ. 44, 464 (2009).

[44] L. Viennot, Spontaneous reasoning in elementary dynamics, Eur. J. Sci. Educ. 1, 205 (1979).

[45] I. Testa, S. Lombardi, G. Monroy, and E. Sassi, An innovative context-based module to introduce students to the optical properties of materials, Phys. Educ. 46, 167 (2011).

[46] A. Colantonio, S. Galano, S. Leccia, E. Puddu, and I. Testa, A teaching module about stellar structure and evolution, Phys. Educ. 52, 015012 (2017).

[47] S. Leccia, A. Colantonio, E. Puddu, S. Galano, and I. Testa, Teaching about mechanical waves and sound with a tuning fork and the Sun, Phys. Educ. 50, 677 (2015).

[48] M. Heritage, Learning Progressions: Supporting Instruction and Formative Assessment (Council of Chief State School Officers, Washington, DC, 2008).

[49] A. Alonzo and J. T. Steedle, Developing and assessing a force and motion learning progression, Sci. Educ. 93, 389 (2009).

[50] V. Kind, Beyond Appearances: Students' Misconceptions about Basic Chemical Ideas, 2nd ed. (School of Education, Durham University, UK, 2004), retrieved on May 08 2017, at http://www.rsc.org/learn-chemistry/resource/ res00002202/beyond-appearances.

[51] A.C. Alonzo and A. Robinson, Developing Learning Progressions for Momentum and Mechanical Energy: Insights for Instruction, Proceedings of the Annual Conference of NARST, San Antonio, TX, 2017.

[52] M. A. Simon and R. Tzur, Explicating the role of mathematical tasks in conceptual learning: An elaboration of the hypothetical learning trajectory, Math. Think. Learn. 6, 91 (2004).

[53] http://www.progettolaureescientifiche.eu/. 